University of Rhode Island

DigitalCommons@URI

Open Access Master's Theses

2013

\title{
LASER-ASSISTED HEATING OF A PLASMONIC NANOFLUID IN A MICROCHANNEL
}

Timothy Walsh

University of Rhode Island, timmiewalsh@gmail.com

Follow this and additional works at: https://digitalcommons.uri.edu/theses

\section{Recommended Citation}

Walsh, Timothy, "LASER-ASSISTED HEATING OF A PLASMONIC NANOFLUID IN A MICROCHANNEL" (2013). Open Access Master's Theses. Paper 155.

https://digitalcommons.uri.edu/theses/155

This Thesis is brought to you for free and open access by DigitalCommons@URI. It has been accepted for inclusion in Open Access Master's Theses by an authorized administrator of DigitalCommons@URI. For more information, please contact digitalcommons-group@uri.edu. 
LASER-ASSISTED HEATING OF A PLASMONIC NANOFLUID IN A MICROCHANNEL

BY

TIMOTHY WALSH

A THESIS SUBMITTED IN PARTIAL FULFILLMENT OF THE REQUIREMENTS FOR THE DEGREE OF

MASTER OF SCIENCE

IN

MECHANICAL ENGINEERING AND APPLIED MECHANICS

UNIVERSITY OF RHODE ISLAND 


\section{MASTER OF SCIENCE THESIS}

OF

\section{TIMOTHY WALSH}

APPROVED:

Thesis Committee:

Major Professor $\quad$ Keunhan Park
DML Meyer
Yana Reshetnyak
Nasser H. Zawia
DEAN OF THE GRADUATE SCHOOL

UNIVERSITY OF RHODE ISLAND 


\begin{abstract}
The work presented in this study analyses the theoretical modeling and experimentation of laser-assisted heating of plasmonic nanofluids (PNFs) in a microchannel for accurate, efficient, and ultra-fast heating of a microdroplet. Suspended plasmonic nanoparticles exhibit strong light absorption and scattering upon the excitation of localized surface plasmons (LSPs), resulting in intense and rapid photothermal heating. Several multiple stepped computational models were utilized to theoretically characterize and verify the laser-assisted heating behavior of gold nanoshells (GNS) and gold nanorod (GNR) plasmonic nanofluid droplets in a microchannel. From the experimental investigation, a full range of controllable steadystate temperatures, room temperature to $100^{\circ} \mathrm{C}$, are confirmed to be achievable for the 780nm-tuned plasmonic nanofluid. Droplet fluid heating is verified to occur as a result of LSP excitation, in time scales of a second, and to be repeatable over many cycles. Additionally, the significance and effects of parameters in the process, such as nanoparticle structure, volumetric concentration, microchannel depth, and laser power density are established. The obtained results in this research may be integrated into other existing microfluidic technologies and biological techniques, such as the polymerase chain reaction, where accurate and ultra-fast heating of microdroplets in a microchannel can greatly improve efficiency.
\end{abstract}




\section{AGKNOWLEDGMENTS}

The research presented in this thesis was conducted at the University of Rhode Island's Micro/Nanoscale Engineering Laboratory under the supervision of Dr. Keunhan Park.

The funding for this was provided by the University of Rhode Island's startup program, a grant from Nation Research Foundation by the Korean Government (NRF2011-220-D00014), and the RI Consortium for Nanoscience and Nanotechnology (RIN2). 


\section{TABLE OF CONTENTS}

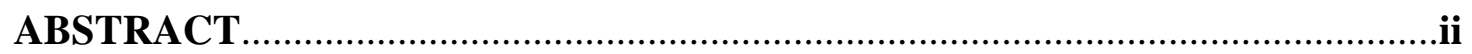

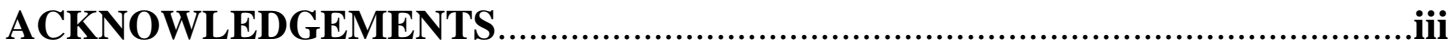

TABLE OF CONTENTS ..............................................................................

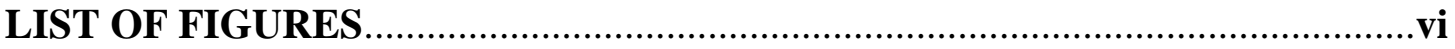

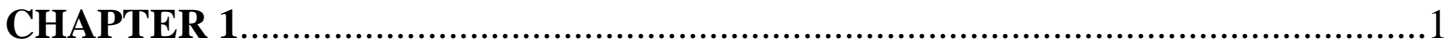

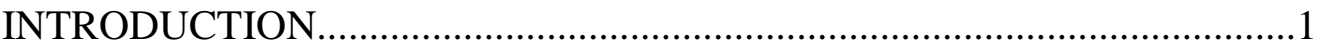

CHAPTER 2

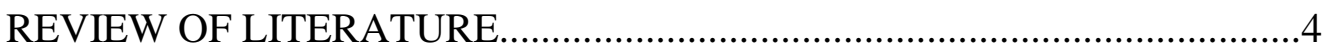

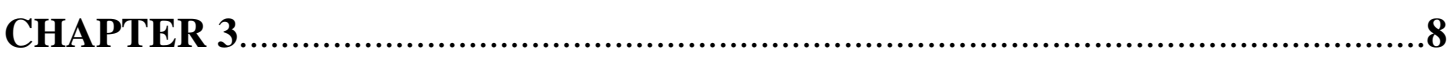

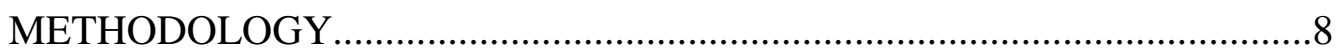

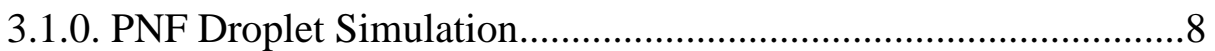

3.1.1 Light Absorption and Scattering............................................

3.1.1.1 Spherical Nanoparticle Case: Mie Solution...........9

3.1.1.2 Rod Nanoparticle Case: BEM Solution................12

3.1.2 Monte Carlo Simulation.....................................................15

3.1.3 Nanoparticle Heating.........................................................18

3.1.3.1 GNS Heating.................................................18

3.1.3.2 GNR Heating...................................................19

3.1.4 FEA Microchannel Modeling..............................................20

3.2.0 PNF Droplet Heating Experimentation.........................................23 
CHAPTER 4 .32

FINDINGS.

4.1 Simulation 32

4.2 Experiment. .35

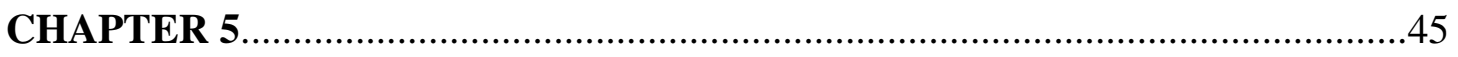

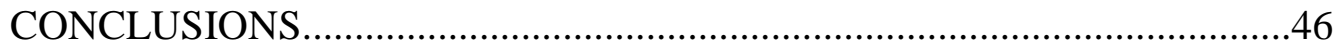

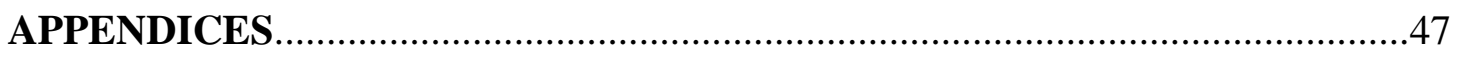

3.1.1.1 Mie Scattering Code.................................................................4 47

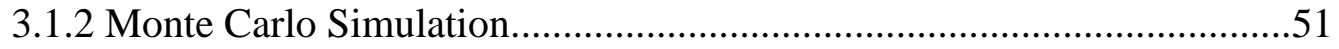

3.1.3.2 GNR Absorption Coefficient Conversion \& Penetration Depth Code.....56

3.1.3.2 Nanoparticle Heating Code.........................................................58

3.1.4 COMSOL FEA Simulation Code...................................................60

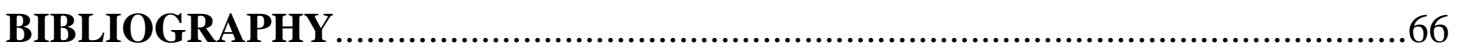




\section{LIST OF FIGURES}

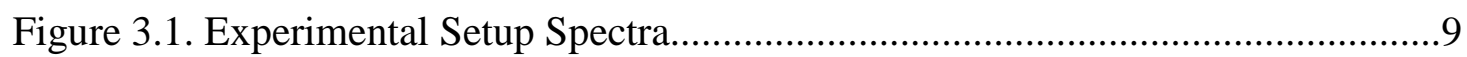

Figure 3.2. Monte Carlo Simulation.........................................................................17

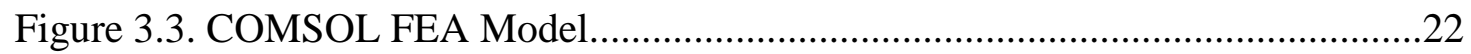

Figure 3.4. Primary Experimental Setup..............................................................23

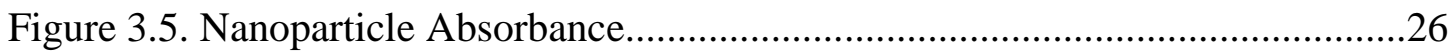

Figure 3.6. Temperature-Fluorescence Setup...........................................................28

Figure 3.7. Droplet Laser Heating..............................................................................

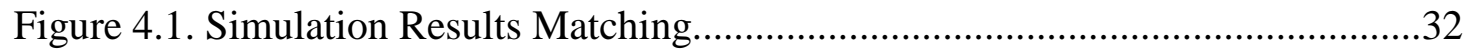

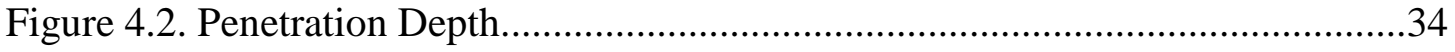

Figure 4.3. Temperature-Fluorescence Relation......................................................36

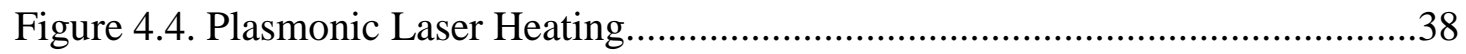

Figure 4.5. Plasmonic Heating Verification...................................................................39

Figure 4.6. Transient Heating Response........................................................................41

Figure 4.7. Transient Thermal Cycling ...................................................................42 


\section{CHAPTER 1}

\section{INTRODUCTION}

Lab-on-a-chip (LOC) technology has led to pivotal progress over the decades in the down-scaling of mechanical fluid handling devices. These microfluidic technologies have been widely utilized across many fields of study in the development of both common and unorthodox applications. Often, many of these same applications require additional heating and cooling processes to be carried out that have been, historically, relatively inefficient and inaccurate [1]. These inefficiencies have existed as a bottleneck in the numerous biological applications specifically catered to by microfluidics. However, the advent of nanotechnology has brought about a surge of new, novel, and ground-breaking improvements on countless hurdles in past technology. One such improvement, where great potential exists for enhanced thermal performance in microfluidic technology, can be easily realized with suspended plasmonic nanoparticles, or plasmonic nanofluids (PNFs)[2].

Plasmonic nanoparticles exhibit strong light absorption upon the excitation of localized surface plasmons (LSP), resulting in efficient, intense, and rapid heating of the PNFs $[\mathbf{2}, \mathbf{3}, \mathbf{4}]$. LSPs result from the confinement of the surface plasmon phenomena in a particle that is on the order of, or smaller than, its own LSP excitation wavelength. Upon LSP excitation, the loosely-bound outer electron charge cloud within the particle

enters an oscillating state, or resonance, resulting from the balancing of the incoming photon energy and the restoring force of the atomic nucleus. The wavelength at which this LSP phenomenon occurs is very highly dependent on several factors such as: 
material, geometry, and local electric fields. For these reasons, plasmonic nanoparticles can be selectively tailored to specific wavelengths of light and thus possess the capacity to be used in a very wide range of applications.[3,4]

One of the microfluidic applications where this enhanced thermal performance from plasmonic heating can be easily outlined, is in the Polymerase Chain Reaction [5]. PCR is a highly utilized biochemical technology to amplify segments of DNA across several orders of magnitude in a relatively short period of time. It has birthed a multi-billion dollar industry and is widely used in many fields of study and applications, such as: virus detection, diseases diagnosis, forensics, vaccine development, etc. [5] The process, itself, involves multiple steps of quick and precise heating and cooling to very specific temperatures over many repeated cycles. Current conventional systems used for PCR can achieve this, albeit through a generally complex and expensive design [1]. These conventional systems typically are equipped with thermoelectric heaters and coolers which generate relatively low heating/cooling rates and non-uniform temperature distributions; resulting in great inefficiencies and thus longer processing times.

It is in applications such as PCR, where the benefits of enhanced thermal performance by optical methods become very clear. Integration of photothermal processes here is highly favorable, as these methods only target the PCR droplets and do not collaterally waste energy and time heating/cooling the LOC device, like the conventional thermoelectric devices. Furthermore, these new photothermal designs possess potential for even faster heating/cooling rates and can improve efficiency still yet[1,4,6,7]. While some other optical heating methods have found success in 
improving LOC technology used for PCR [8-12], plasmonic heating still offers equal or greater potential.

This work specifically aims to demonstrate, both computationally and experimentally, the laser-assisted heating of a plasmonic nanofluid microdroplet in a microchannel for controllable, accurate, rapid, and effective heating of the medium. From this investigation, we anticipate to find a full range of controllable temperatures, room temperature to boiling, to be achievable for a 780-nm-tuned PNF. We expect to effectively verify that this fluid heating truly occurs as a result of LSP excitation, in time scales of milliseconds, and to be fully repeatable over time. Additionally, we will verify the importance and effects of parameters in the process, such as volumetric concentration, microchannel depth, and laser power density. The obtained results in this research will be evaluated for their viability of integration into other existing microfluidic technologies and biological techniques, such as the Polymerase Chain Reaction, where quick and accurate heating may greatly improve efficiency. 


\section{CHAPTER 2}

\section{REVIEW OF LITERATURE}

Accurately heating the fluids within microfluidic devices in a controllable manner is an extremely important topic in many LOC-based applications. These processes may require temperature homogeneity or gradients for their specific purposes, however both cases aim for the highest accuracy and repeatability as possible. Notable applications that heavily rely on this features of microfluidics in the areas of physics, chemistry, and biotechnology are: Polymerase Chain Reaction (PCR) [13-16], Temperature Gradient Focusing for Electrophoresis (TGF) [17], protein crystallization [18], solution mixing [19], and optofluidic guidance[20]. While many various heating techniques have emerged in recent years to improve these applications, they each demonstrate different advantages and drawbacks when concerned with terms of accuracy, cost, ease of integration, type of control, etc.

The vast majority of microfluidic heating or cooling methods are carried out through contact-based external elements such as Joule heaters or Peltier devices. Often these elements are bonded directly to the surface of the microfluidic chip, but are sometimes embedded within the device as well. Contemporary contact-based microfluidic $[\mathbf{2 1}, \mathbf{2 2}, \mathbf{1 6}]$ and capillary-action devices [15] developed for batch PCR applications achieve maximum heating/cooling ramp rates of $\sim 10^{\circ} \mathrm{C} / \mathrm{sec}[\mathbf{2 3}, \mathbf{2 4}]$. Some claims to ramp rates as high as $30-40^{\circ} \mathrm{C} / \mathrm{sec}$ have been made, albeit through highly complicated channel designs, utilizing micro-embedded elements with low yields 
$[\mathbf{2 1}, \mathbf{1 4 , 2 5}]$. Furthermore, the common practice of using PDMS substrates tends to cause heating/cooling inefficiencies as its thermal conductivity rests relatively low.

Conversely, there are a number of studied and utilized optical methods in microfluidic heating and cooling as well. These noncontact-based techniques are often considered preferable as they generally aim to heat only the fluid inside rather than an external device and dissipate heat outward into the medium. This is highly advantageous, because resources are used more efficiently in these processes by using less energy and time that would otherwise, conventionally, be wasted in heating/cooling the microfluidic device as well. It has been found that when compared to conventional techniques, enhanced thermal cycling and faster reaction rates can be achieved [1].

The first of these methods is microwave dielectric heating with time-varying electric fields (from 3-20 GHz) [10-12]. It can be designed to be spatially selective thanks to miniaturized elements and waveguide configurations and has been shown to be successful in PCR [10] and heating of biological cells [12]. Microwave heating methods have so far found best use in PCR applications when compared to conventional techniques when working with volumes of $2.5-15 \mathrm{~mL}$. It is at this scale, with reduced surface area to volume ratios, that conventional techniques greatly lose their efficiency.

A second optical method that has been utilized is infrared laser heating using aqueous solutions [8,9]. This method has gained interest because of the cheap cost and reliability of the modern laser diode; its the extremely small spatial resolution and 
highly precise control as a heat source is very well suited to even the smallest droplet sizes employed in microfluidic devices. Kim et al. [8] found that a de-focused $1.46 \mu \mathrm{m}$ wavelength laser diode could be utilized to heat nanoliter-sized liquid water droplets with sub- $1^{\circ} \mathrm{C}$ accuracy in order to successfully perform a real-time PCR. This wavelength was chosen ad hoc as water absorbs extremely strongly in this specific infrared spectrum.

The third optical method that has been pursued for use in microfluidics is the utilization of the specialized nanoparticles to enhance optical heat generation. This method can further be divided into two sub-categories: non-plasmonic and plasmonic heating. Non-plasmonic heating is very simply achieved through broad absorption of incident light from a highly energetic source (i.e. laser); carbon nanoparticle structure mixtures are most exemplary in this sub-category. Many studies have found that these mixtures are very efficient photothermal converters, especially keen for solar-thermal applications [26,27]. Additionally, exploitation of the enhancing effects on the effective fluid thermal conductivity [28] by noble metal nanoparticle mixtures have also been successfully employed in improving PCR efficiencies [29, 30]. However, these enhanced thermal conductivity effects only typically become considerable at volumetric concentrations of $>1 \%$, which can be very difficult or costly to manufacture for many nanoparticle structures.

Plasmonic heating is achieved through the excitation of LSPs in plasmonic nanoparticles, typically by laser. The heat produced from this phenomenon can be extremely intense, rapid, and localized $[4,6,7]$; reaching electron temperatures at the nanoparticle surface as high as $1270 \mathrm{~K}$ in tens of nanoseconds have been recorded 
$[31,7]$. Commonly in excessively irradiated cases, localized boiling has been observed in the surrounding sub-cooled fluid medium; as well as micro-thermophoresis [26] and nanoparticle deformation[4,31]. While many nanoparticle materials (i.e. noble metals) and geometries exhibit LSPs, silver and gold nanoparticle structures have been found particularly favorable in biological LOC-based applications because of their good biocompatibility, chemical stability, and easily tunable excitation wavelengths within the visible spectrum.

Plasmonic nanoparticles have found specific use in microfluidic applications for improved mixing techniques [32], optically driven fluid guidance and pumping in a microchannel $[\mathbf{2 0 , 3 3}]$, refractive index detective sensor [34], and particle trapping and manipulation $[\mathbf{3 4 , 3 5}]$. Nevertheless, very little work has been significantly developed in the vein of controlled and accurate plasmonic heating of individual microdroplets in a microchannel. Furthermore, while there have been great effort towards analytical and computational approaches to plasmonic nanoparticle behavior [36-41], very little attention has been given towards pairing these solutions with FEA simulation and comparing with experimental results. 


\section{CHAPTER 3}

\section{METHODOLOGY}

\subsubsection{PNF Droplet Simulation}

\subsubsection{Light Absorption and Scattering}

The first steps aims to identify the useable wavelengths of light in the proposed experimental setup. This is important, as different materials provide various bands of light absorption and may cause difficulties in allowing the intended light to reach its intended target. The absorption spectrum of common and likely materials in a simple microchannel setup were plotted in Figure 1a. Common microchannel materials considered for are polydimethylsiloxane (PDMS) and borosiliicate glass. The microchannel fluids considered are water and mineral oil. Figure 1a shows that it was found that the range from $500-1000 \mathrm{~nm}$ is the most promising optical window for experimentation, as virtually no light absorption is expected for any of these materials. Therefore, the QD fluorescence excitation and emission, as well as the LSPR wavelength should fall within this spectrum without significant overlapping (Figure 1b). 


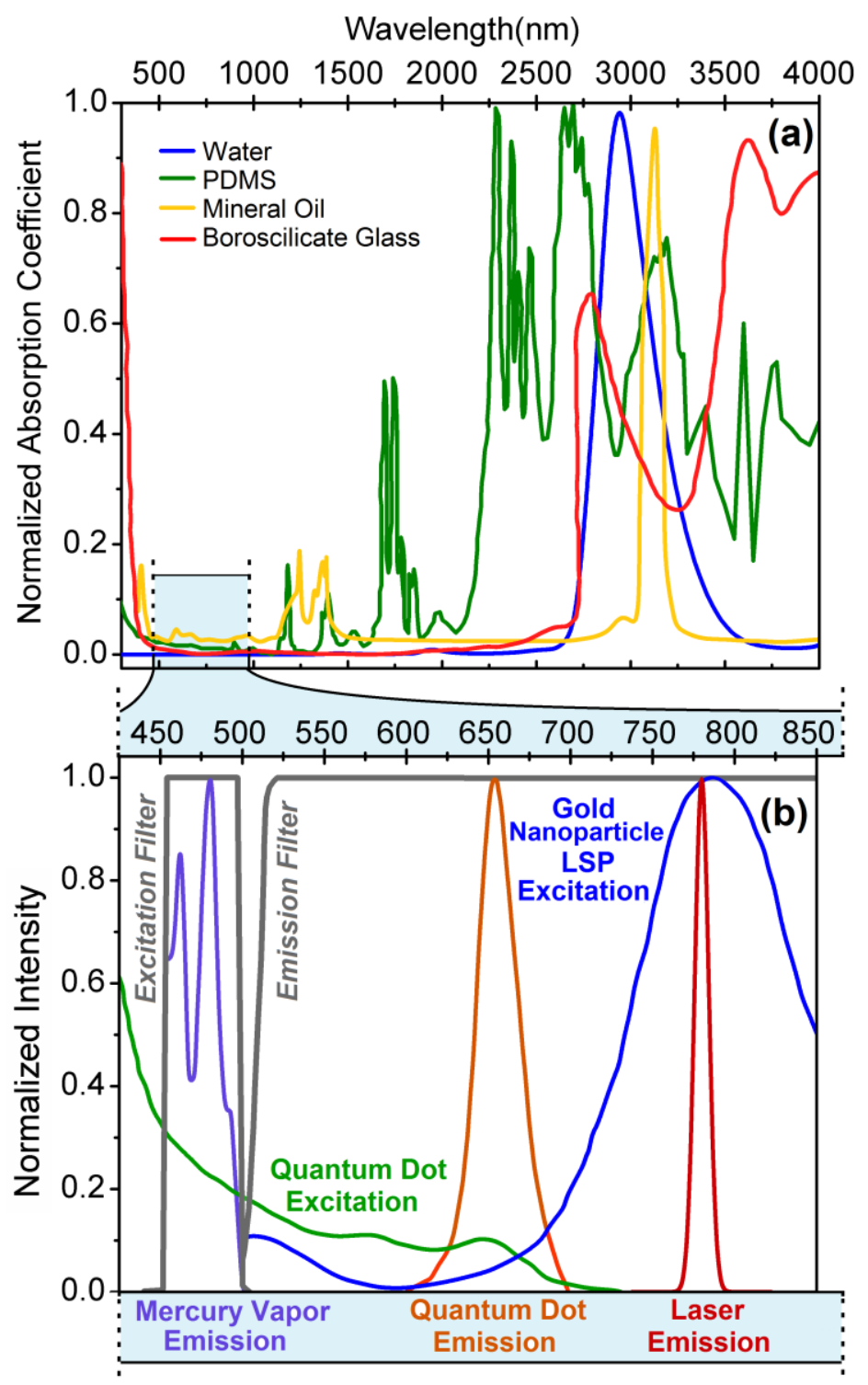

Figure 3.1 Experimental Setup Spectra - (a) Demonstrates the transmissible wavelengths for experimentation after overlaid absorption spectra from microchannel materials. (b) Normalized diagram of excitation and emission spectra confined by filter bands utilized in the experimental setup. 


\subsubsection{Spherical Nanoparticle Case: Mie Solution}

A Mie scattering theory was numerically calculated $[2,42]$ to identify and consider the absorption and scattering coefficients of commonly available plasmonic spherical nanoparticles (aluminium, silver, and gold) of various dimensions and concentrations. The Mie scattering formulation is a specific set of solutions to Maxwell's equations in spherical coordinates and thus best suited for isotropic, homogenous, dielectric spherical particles whose size is comparable to that of the particular incident wavelength of light. This far-field solution of the Mie scattering theory is realized in terms of two scattering functions,

$$
\begin{aligned}
& S_{1}(\theta)=\sum_{n=1}^{\infty} \frac{2 n+1}{n(n+1)}\left[a_{n} \pi_{n}(\cos \theta)+b_{n} \tau_{n}(\cos \theta)\right] \\
& S_{2}(\theta)=\sum_{n=1}^{\infty} \frac{2 n+1}{n(n+1)}\left[b_{n} \pi_{n}(\cos \theta)+a_{n} \tau_{n}(\cos \theta)\right]
\end{aligned}
$$

where $\theta$ is the scattering angle. The functions $\pi_{n}$ and $\tau_{n}$ are given by,

$$
\begin{aligned}
& \pi_{n}(\cos \theta)=\frac{1}{\sin \theta} P_{n}^{1}(\cos \theta) \\
& \tau_{n}(\cos \theta)=\frac{d}{d \theta} P_{n}^{1}(\cos \theta)
\end{aligned}
$$

where $P_{n}^{1}$ are first-order Legendre polynomials. However, the prime effort in finding the solution to the Mie formulation is determining the coefficients $a_{n}$ and $b_{n}$,

$$
a_{n}=\frac{\Psi_{n}^{\prime}(m \chi) \Psi_{\mathrm{n}}(\chi)-\mathrm{m} \Psi_{\mathrm{n}}(\mathrm{m} \alpha) \Psi_{\mathrm{n}}^{\prime}(\chi)}{\Psi_{\mathrm{n}}^{\prime}(\mathrm{m} \chi) \xi_{\mathrm{n}}(\chi)-\Psi_{\mathrm{n}}(\mathrm{m} \chi) \xi_{\mathrm{n}}^{\prime}(\chi)}
$$




$$
b_{n}=\frac{m \Psi^{\prime}{ }_{n}(m \chi) \Psi_{n}(\chi)-\Psi_{n}(m \chi) \Psi_{n}^{\prime}(\chi)}{m \Psi_{n}^{\prime}(m \chi) \xi_{n}(\chi)-\Psi_{n}(m \chi) \xi_{n}^{\prime}(\chi)}
$$

where $\chi=\left(2 \pi r_{o} n_{w}\right) / \lambda$ is the size parameter of particle outer radius $\left(r_{o}\right)$ and index of refraction of water $\left(n_{w}\right), \mathrm{m}$ is the index of refraction of the particle medium, and $\Psi_{\mathrm{n}}$ and $\xi_{\mathrm{n}}$ are related to spherical Bessel functions.

From these equations, the scattering and absorption cross-sections, and the asymmetry parameter, respectively, can be expressed in terms of $a_{n}$ and $b_{n}$.

$$
\begin{gathered}
C_{s}=\frac{2 \pi}{x^{2}} \sum_{n=1}^{\infty}(2 n+1)\left(\left|a_{n}\right|^{2}+\left|b_{n}\right|^{2}\right) \\
C_{a}=\frac{2 k}{x^{2}} \sum_{n=1}^{\infty}(2 n+1) \mathfrak{R}\left(a_{n}+b_{n}\right)-C_{s} \\
g=2 \sum_{n=1}^{\infty}\left[\frac{n(n+2)}{n+1} \mathfrak{R}\left(a_{n} a_{n+1}^{*}+b_{n} b_{n+1}^{*}\right)+\frac{2 n+1}{n(n+1)} \mathfrak{R}\left(a_{n}+b_{n}^{*}\right)\right]
\end{gathered}
$$

Commonly, these cross-section values are divided by the geometric cross-sectional area to give dimensionless scattering/absorption efficiency parameters, $Q_{s, a}=$ $C_{s, a} / \pi r^{2}$. However, when dispersed nanoparticles are taken into consideration rather than single nanoparticles, a more meaningful parameter is realized in the scattering and absorption coefficients, $\sigma$ and $\alpha$,

$$
\begin{gathered}
\sigma_{\lambda}=\sum_{i=1}^{n} \frac{3 f_{i}}{2 D_{i}} Q_{s, i} \\
\alpha_{\lambda}=\left(1-\sum_{i=1}^{n} f_{i}\right) \alpha_{H_{2} O, \lambda}+\sum_{i=1}^{n} \frac{3 f_{i}}{2 D_{i}} Q_{a, i}
\end{gathered}
$$


where $\alpha_{\mathrm{H}_{2} \mathrm{O}, \lambda}$ is the absorption coefficient of water and $f$ is the volume fraction of the mixed nanoparticles. The coefficients can be generally expressed as summations in order to account for a case of multiple different nanoparticle structures in the mixture.

During this calculation process, consideration was given to the peak absorption wavelength and practicality of particle size in order to effectively optimize the 1000nm limited spectrum window available for the LSPR excitation, QD excitation, and QD emission without significant overlaps. From this process and with these restrictions, the best suited spherical nanoparticles in this study were determined to be silica-core gold nanoshells.

\subsubsection{Rod Nanoparticle Case: BEM Solution}

Since the Mie scattering theory is only valid for spherical and elliptical particles, a boundary element method, or BEM, program called MNPBEM [43,44] was utilized to calculate the absorption cross-sections of GNR structures. BEM is a mathematically rigorous numerical computational method for solving the full microscopic Maxwell equations in terms of surface integrals of arbitrarily shaped dielectric bodies with homogenous dielectric properties. The boundary element method works by constructing a mesh over the modeled surface and aims to calculate the charges and currents that act as the sources of the induced electromagnetic fields at interfaces separating different media.

Beginning with the microscopic formulation of Maxwell's equations in frequency domain $\omega$, 


$$
\begin{array}{ll}
\boldsymbol{\nabla} \cdot \overrightarrow{\boldsymbol{D}}=4 \pi \rho & ; \quad \boldsymbol{\nabla} \times \overrightarrow{\boldsymbol{H}}+i k \overrightarrow{\boldsymbol{D}}=\frac{4 \pi}{c} \boldsymbol{j} \\
\boldsymbol{\nabla} \cdot \overrightarrow{\boldsymbol{B}}=0 & ; \quad \boldsymbol{\nabla} \times \overrightarrow{\boldsymbol{E}}-i k \overrightarrow{\boldsymbol{B}}=0
\end{array}
$$

where $k=\omega / c$ is the wavenumber of light in vacuum, $\overrightarrow{\boldsymbol{D}}=\epsilon \overrightarrow{\boldsymbol{E}}$ is the electric displacement, $\overrightarrow{\boldsymbol{B}}=\mu \overrightarrow{\boldsymbol{H}}$ is the magnetic induction, $\epsilon(\overrightarrow{\boldsymbol{r}}, \omega)$ is the electrical permitivity (or dielectric function), and $\mu(\overrightarrow{\boldsymbol{r}}, \omega)$ is the magnetic permeability; both the electric and magnetic fields $\overrightarrow{\boldsymbol{E}}$ and $\overrightarrow{\boldsymbol{B}}$ can be then expressed in terms of a scalar and vector potential $\phi$ and $\overrightarrow{\boldsymbol{A}}$, respectively as,

$$
\overrightarrow{\boldsymbol{E}}=i k \overrightarrow{\boldsymbol{A}}-\nabla \phi \quad ; \quad \overrightarrow{\boldsymbol{H}}=\frac{1}{\mu} \boldsymbol{\nabla} \times \overrightarrow{\boldsymbol{A}}
$$

By way of the Lorentz gauge condition, the relation $\boldsymbol{\nabla} \cdot \overrightarrow{\boldsymbol{A}}=i k \epsilon \phi$ can be adopted; and the general solution form of these two equations, for $\overrightarrow{\boldsymbol{r}}$ inside a medium $j$, can be written as,

$$
\phi(\overrightarrow{\boldsymbol{r}})=\frac{1}{\epsilon_{j}(\omega)} \int d \overrightarrow{\boldsymbol{r}^{\prime}} G_{j}\left(\left|\overrightarrow{\boldsymbol{r}}-\overrightarrow{\boldsymbol{r}}^{\prime}\right|\right) \rho\left(\overrightarrow{\boldsymbol{r}}^{\prime}\right)+\int_{S_{j}} d \overrightarrow{\boldsymbol{s}} G_{j}(|\overrightarrow{\boldsymbol{r}}-\overrightarrow{\boldsymbol{s}}|) \sigma_{j}(\overrightarrow{\boldsymbol{s}})
$$

and

$$
\overrightarrow{\boldsymbol{A}}(\overrightarrow{\boldsymbol{r}})=\frac{\mu_{j}(\omega)}{c} \int d \overrightarrow{\boldsymbol{r}}^{\prime} G_{j}\left(\left|\overrightarrow{\boldsymbol{r}}-\overrightarrow{\boldsymbol{r}}^{\prime}\right|\right) \overrightarrow{\boldsymbol{J}}\left(\overrightarrow{\boldsymbol{r}}^{\prime}\right)+\int_{\boldsymbol{S}_{j}} d \overrightarrow{\boldsymbol{s}} G_{j}(|\overrightarrow{\boldsymbol{r}}-\overrightarrow{\boldsymbol{s}}|) \overrightarrow{\boldsymbol{h}}_{j}(\overrightarrow{\boldsymbol{s}})
$$

where $S_{j}$ denotes the boundary of medium $j=1,2$ and $G_{j}$ is the Green function of the wave equation,

$$
G_{j}(r)=\frac{e^{i k_{j} r}}{r}
$$


where $k_{j}=k \sqrt{\epsilon_{j} \mu_{j}}$. After rigorous employment of the boundary conditions to Maxwell's equations in order to calculate the continuity of surface charges and currents, the working equations for nonmagnetic materials $\left(\mu_{j}=1\right)$ can be derived to the following,

$$
\begin{aligned}
\sigma_{j}=G_{j}^{-1} \Sigma^{-1}\left\{D^{e}-\epsilon_{j^{\prime}} \Sigma_{j^{\prime}} \phi^{e}+i k \boldsymbol{n}_{s} \cdot \Delta^{-1}\left[\left(\epsilon_{1}-\epsilon_{2}\right)\right]\left(\vec{\alpha}+i k \boldsymbol{n}_{s} \epsilon_{j^{\prime}} \phi^{e}\right)\right. \\
\left.\left.+\left(\epsilon_{2} \Sigma_{1}-\epsilon_{1} \Sigma_{2}\right) \overrightarrow{\mathbf{A}}^{\mathrm{e}}\right]\right\}
\end{aligned}
$$

and

$$
\overrightarrow{\boldsymbol{h}}_{\boldsymbol{j}}=G_{j}^{-1} \Delta^{-1}\left[\vec{\alpha}-\Sigma_{j} \overrightarrow{\boldsymbol{A}}^{e}+i k \boldsymbol{n}_{s}\left(G_{1} \epsilon_{1} \sigma_{1}-G_{2} \epsilon_{2} \sigma_{2}\right)\right]
$$

where

$$
\begin{gathered}
\phi^{e}=\phi_{2}^{e}-\phi_{1}^{e} \quad, \quad \overrightarrow{\boldsymbol{A}}^{e}=\overrightarrow{\boldsymbol{A}}_{2}^{e}-\overrightarrow{\boldsymbol{A}}_{1}^{e}, \\
\Sigma_{j}=H_{j} G_{j}^{-1} \quad, \quad \Delta=\Sigma_{1}-\Sigma_{2} \\
\Sigma=\epsilon_{1} \Sigma_{1}-\epsilon_{2} \Sigma_{2}+k^{2}\left(\epsilon_{1}-\epsilon_{2}\right)^{2} \boldsymbol{n}_{s} \cdot \Delta^{-1} \boldsymbol{n}_{s}
\end{gathered}
$$

From the discretization of these equations, the resulting surface charges and currents at the inside and outside of the particle boundaries are finally computed.

On the user end, the program requires several initial parameters for successful computation including: generic geometric shape, particle dimensions, indices of refraction for all media, light spectrum, and light vector and polarization values. In the case of the GNR structures, the initial parameters were specified as: symmetric 
cylinder with hemispherical caps, $10 \mathrm{~nm}$ X $38 \mathrm{~nm}$, refractive indices for gold and water, $785 \pm 10 \mathrm{~nm}$, and circularly polarized light both parallel and perpendicular to the particle axis. Ultimately, the boundary element method utilized here is able to compute the effective particle absorption and scattering cross-sections of the individual GNR. However, because the GNR is not symmetric in all spatial dimensions, it exhibits different absorption and scattering behavior in various orientations relative to the incident light. For this reason, two BEM computations were done for the GNR case: first oriented perpendicular to the light vector and second oriented parallel with. The scattering cross-sections for these GNR structures were found to be significantly smaller (greater than 1 order of magnitude) in comparison to their absorption cross-sections; thusly, the effects of light scattering were assumed negligible in subsequent calculations.

\subsubsection{Monte Carlo Simulation}

In the case of the GNS-suspended plasmonic nanofluids, the light scattering becomes comparable to the light absorption when the LSP is excited. Therefore, it cannot be neglected in determining the heat absorption behavior and the complicated scattering phenomena should be fully taken into consideration. A Monte Carlo algorithm [46] was employed to solve the radiative transport equation [47] with consideration of the volume scattering from the GNSs. This code was used to track the probabilistic 2-dimensions paths and occurrences of individual photon bundles entering perpendicular to the microchannel top, through the droplet, and exiting the microchannel bottom. The 3rd dimension, the length of the channel, in this code was 
assumed to be infinite for the microchannel and droplet in order to greatly simply the formulation.

In the Monte Carlo simulation, the "drift" process (during which the photon bundle propagates) is treated independently from the scattering process (during which the photon bundle changes its propagation direction). It is assumed that the drift process is followed by the scattering process based on the scattering probability $P_{\text {scat }}=1-\exp \left(-L \sigma_{\lambda}\right)$ where $L$ is the propagation length of phonon bundle calculated by $L=-\ln \left(R_{1}\right) /\left(\sigma_{\lambda}+\alpha_{\lambda}\right), \sigma_{\lambda}$ is the scattering coefficient of the GNS, and $\alpha_{\lambda}$ is the combined absorption coefficient of the GNS and water. A uniform random number $R_{1}$ is generated to satisfy $0 \leq R_{1} \leq 1$ by a quasi-random sequence. It should be noted that the estimated L stochastically represents the mean-free-path (i.e., averaged propagation length) of $1 /\left(\sigma_{\lambda}+\alpha_{\lambda}\right)$. In determining whether to accept or reject the scattering event, an addiontional uniform random number $R_{2}$ is generated and compared with $P_{\text {scat }}$; if the value of $R_{2} \leq P_{\text {scat }}$, then the ray will be scattered at the next nanoparticle encounter. Upon the scattering process, the post-scattering direction of the photon bundle is expressed by the polar angle and the azimuth angle with respect to the pre-scattering direction vector. For the greater simplicity, the polar angle is also stochastically determined by transforming the Henyey-Greenstein $(\mathrm{H}-\mathrm{G})$ scattering phase function. The azimuthal angle is determined by assuming that the scattering is isotropic in the azimuthal direction. It should be noted that the $\mathrm{H}-\mathrm{G}$ scattering phase function is an approximation and cannot be regarded as the rigorous phase function for the light scattering from the GNS. 
Despite this, the $\mathrm{H}-\mathrm{G}$ scattering phase function provides the probability density of light scattering that can be seamlessly integrated with the Monte Carlo simulation.

Although the absorption of the GNS-based PNF is mainly due to the plasmonic absorption by the nanoparticles, the Monte Carlo simulation treats the whole of the PNF as an effective medium in which the energy of the photon bundle uniformly reduces by a factor of $e^{-\alpha_{\lambda} L}$ due to volumetric absorption while traveling a distance $L$. In the event that the photon bundle reaches the bottom surface of the glass, there is no consideration for reflection back into the effective medium. The drift and scattering processes described are repeated until the photon bundle leaves the microchannel or 99.9\% of its energy is absorbed inside the PNF medium.

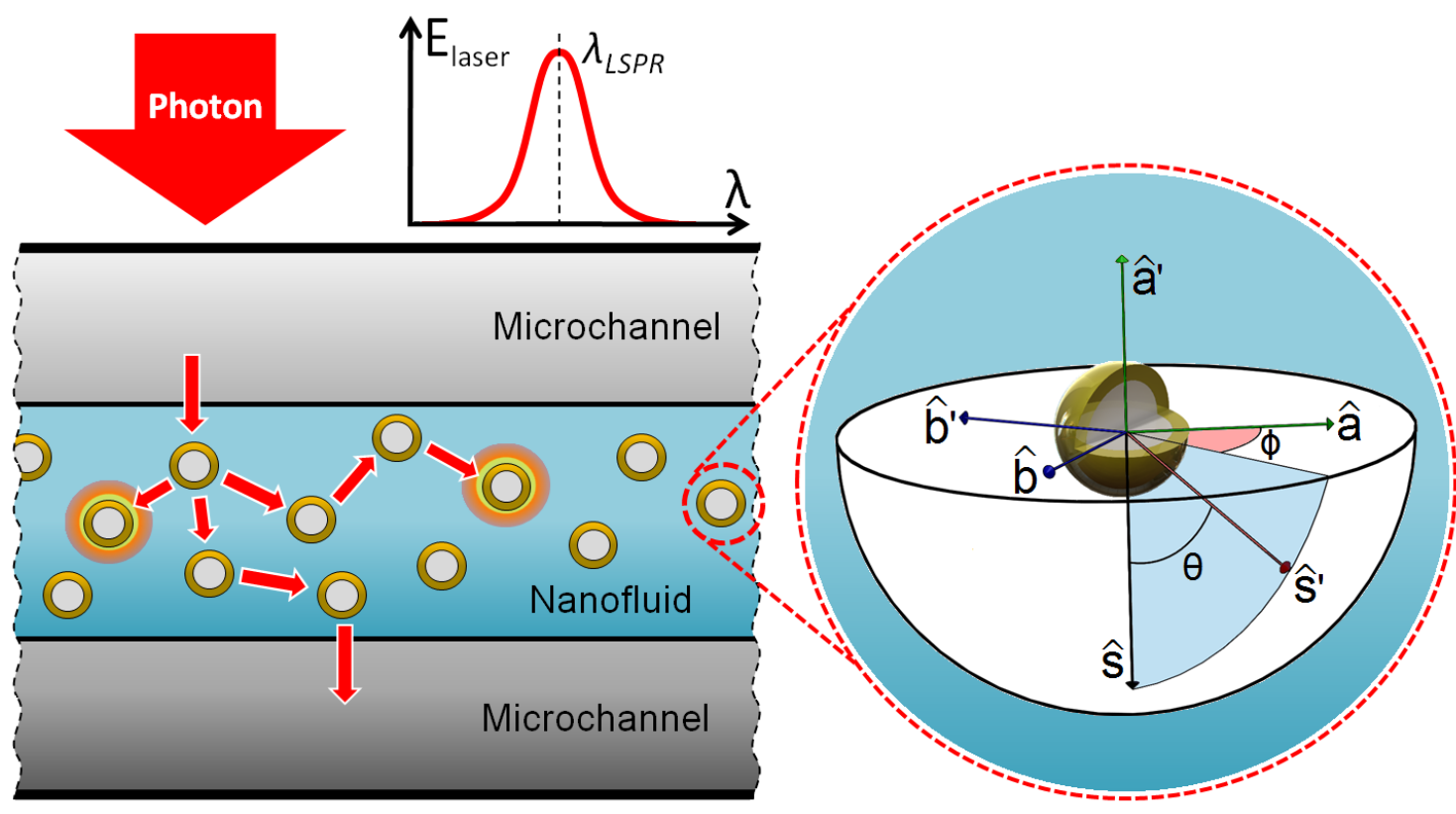

Figure 3.2 Monte Carlo Simulation - Diagram of the methodology employed during the Monte Carlo simulation. 
All Monte Carlo simulations were run for 20 million photon bundle cases in wavelength increments appropriately distributed by the standard deviation of the full width half maximum spectrum, (FWHM) of the $785 \mathrm{~nm}$ laser. The results of the 20 million iterations were exported as a series of data files for the spectral absorptance of each wavelength increment and across the predefined increments of the total droplet depth.

\subsubsection{Nanoparticle Heating}

\subsubsection{GNS Heating}

Determining the amount of heat generated by each GNS-based PNF droplet case as a result of the photon absorption was the next step taken. A script in Matlab was written to import and extract the spectral absorptance distribution data outputted by the previous Monte Carlo simulation step, as well as the initial parameters defined during the Mie scattering solution. First, the incident laser power, $P_{\text {inc }}$, at the top surface of the droplet was calculated from spectral irradiance power density of the laser as,

$$
P_{i n c}(\lambda)=I_{i n c}^{\prime \prime}(\lambda) \tau_{g}=\frac{-\tau_{g}\left(\lambda-\lambda_{o}\right)^{2}}{\frac{2 \varsigma^{2}}{\varsigma \sqrt{2 \pi}}}
$$

where $I_{i n c}^{\prime \prime}$ is the incident spectral intensity flux, $\tau_{g}$ is the light transmittance of the upper medium in the microchannel, $\lambda_{o}$ is laser peak wavelength, and $\zeta$ is the standard deviation with respect to the laser FWHM spectrum. Next, $P_{\text {inc }}$ was multiplied through with the spectral absorptance depth distribution and integrated over the full 
laser spectrum; giving way to the volumetric laser energy absorption rate, $\dot{q}_{t o t}$ at each droplet depth increment,

$$
\dot{q}_{\text {tot }}(z)=\frac{d}{d z} \int_{\lambda_{\min }}^{\lambda_{\max }} A_{\lambda}(\lambda, z) P_{\text {inc }}(\lambda) d \lambda
$$

where $A_{\lambda}$ is the spectral absorptance depth distribution, and $\lambda_{\min , \max }=\lambda_{o} \pm 5 \varsigma$. The results of this calculation were exported as a single data file for the volumetric heat generation across the predefined increments of the total droplet depth.

\subsubsection{GNR Heating}

Determining the amount of heat generated by the GNR-based nanofluid was approached differently, because use of the Monte Carlo algorithm was restricted to spatially symmetric particles. A Matlab script was used to approximately convert the axial and lateral spectral absorption cross-sections to the averaged effective spectral absorption coefficient. By considering random particle orientation in 3 dimensions, the effective absorption cross-section at each wavelength increment can be written as,

$$
C_{e f f}(\lambda)=\frac{1}{\pi / 2} \int_{0}^{\pi / 2}\left[C_{a}(\lambda) \cos (\theta)+C_{\ell}(\lambda) \sin (\theta)\right] d \theta
$$

where $C_{a}$ and $C_{\ell}$ are the axial and lateral absorption cross-sections, respectively. The function was integrated only up to $\pi / 2$ as further rotation beyond this angle would be redundant due to particle symmetry. The, effective spectral absorption cross-section values were multiplied by the number density $(N)$ of the GNR mixture to obtain the effective spectral absorption coefficients, 


$$
\alpha_{\lambda}(\lambda) \cong N C_{e f f}(\lambda)
$$

Using the obtain spectral absorption coefficient, the Beer-Lambert Law was employed to acquire the spectral penetration depth from,

$$
P(\lambda, z)=P_{i n c}(\lambda) e^{-\alpha_{\lambda}(\lambda) z}
$$

where $P_{\text {inc }}$ is the incident laser power and $z$ is the droplet depth. It should be noted that the spectral penetration depth can be expressed as $\delta_{p}(\lambda)=\frac{1}{\alpha_{\lambda}(\lambda)}$ or by defining the depth at which the spectral light intensity has decayed to $e^{-1}$ of its incident value. Since the GNR scattering effects are negligible, the spectral penetration depth values, can be considered to be a reasonable approximation of the spectral absorptance $\left(A_{\lambda}\right)$ across the total droplet depth. To this end, the volumetric laser energy absorption rate, $\dot{q}_{t o t}$ could then be calculated the same way as that of the GNS case.

\subsubsection{FEA Microchannel Modeling}

To compute the thermal behavior of the microchannel systen upon photothermal heating, a number of finite element analysis (FEA) models were developed with a commercially available software package: COMSOL Multiphysics 3.5a. The iterative solver utilized by this program can handle the complex calculations needed to solve both the steady-state and transient conduction heat equations in three dimensions. The developed FEA model was considered as a symmetric rectangular quarter of the microchannel system in order to reduce the rigor of computation without detrimentally effecting solution accuracy. Two distinct steady-state and transient models were constructed to study the behavior of the microchannel system under the 
COMSOL general heat transfer module. The effects of natural convection were not considered in this FEA model, as the Rayleigh number was calculated to be $<0.1$ indicating the conduction was the primary transfer of heat in this system. Furthermore, the effects of internal convection within an aqueous microdroplet of such small volume would produce negligible results on the overall heating behavior of the microchannel system. For these reasons, only heat conduction was considered in the FEA models and all sub-domains were treated as effective solids.

Figure 3.3a shows the designed FEA model dimensions and subdomain conditions. It should be noted that the PNF and oil sub-domains were assumed and modeled as rectangular droplets; with the same cross-section dimensions as the microchannel. Additionally, the thermal properties of the PNF and adjacent oil phase were defined as that of pure liquid water and of mineral oil USP, respectively. The encompassing microchannel sub-domain was defined with the thermal material properties of common PDMS media.

In order to simulate the plasmonic heating behavior that occurs during laser irradiation, the PNF sub-domain was divided into finite slices along the z-axis and parallel to the xy-plane of prescribed thicknesses. Specific discretization of the PNF sub-domain depth was delineated by importing the volumetric heat generation result detailed in the previous sections. Figure 3.3b depicts a visual example of the methodology employed during this step. Each slice was treated as temporary subdomain defined with its respective calculated volumetric heat generation term. COMSOL could solve the FEA model for either its steady-state or transient temperature solution. As shown in Figure3.3c, both COMSOL models were solved 
using a total of 2807 tetrahedral meshing elements, with an increased refinement about the PNF sub-domain, and a total of 106729 degrees of freedom.

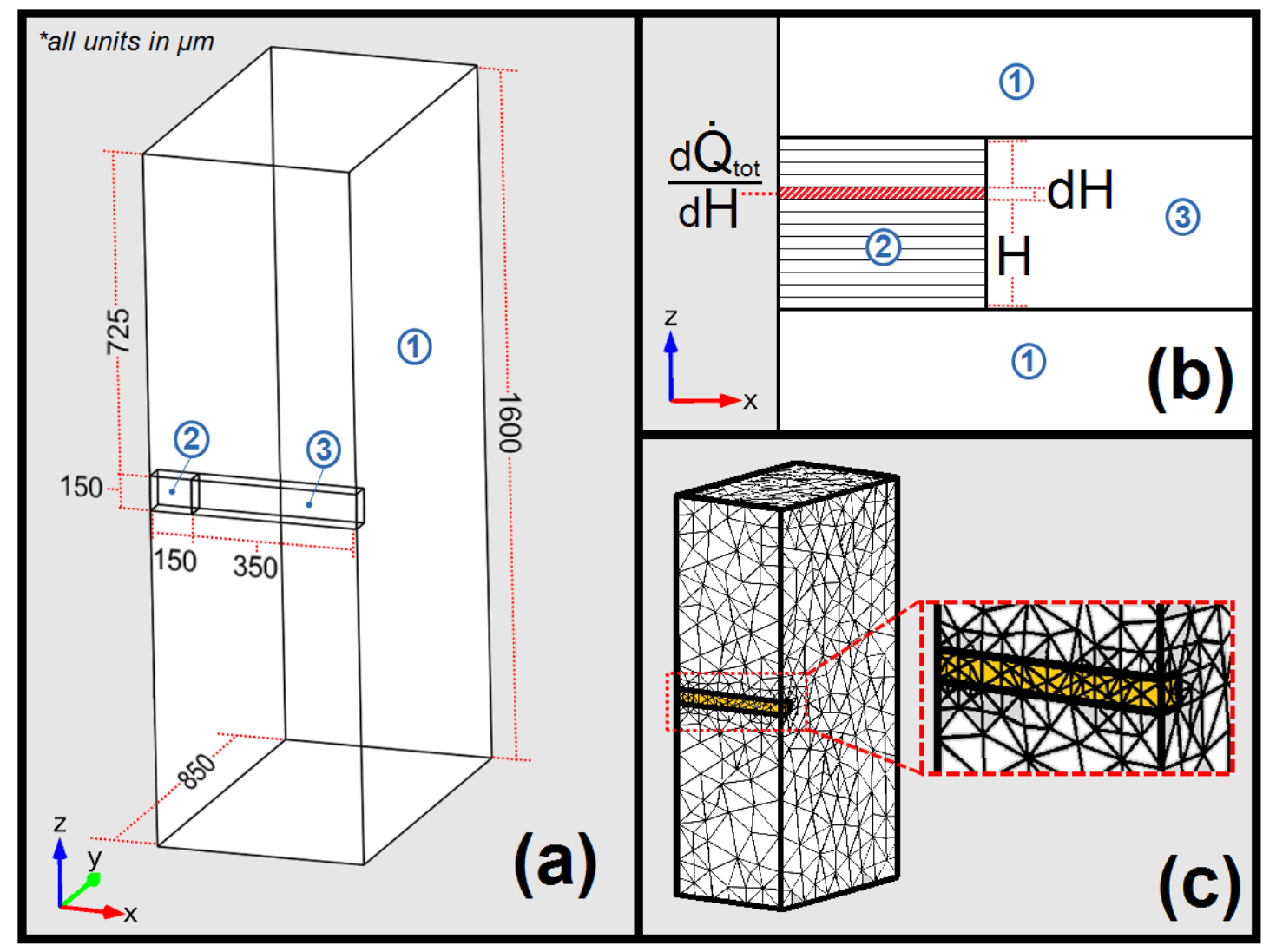

Figure 3.3 COMSOL FEA Model - (a) Full FEA model in COMSOL with dimensions and subdomains: PDMS, water, and mineral oil USP labeled 1,2,3 respectively. (b) The discretization process of the volumetric heat generation in the microdroplet. (c) Fully meshed FEA model in COMSOL. 


\subsubsection{PNF Droplet Heating Experimentation}

\subsubsection{Primary Optical Setup}

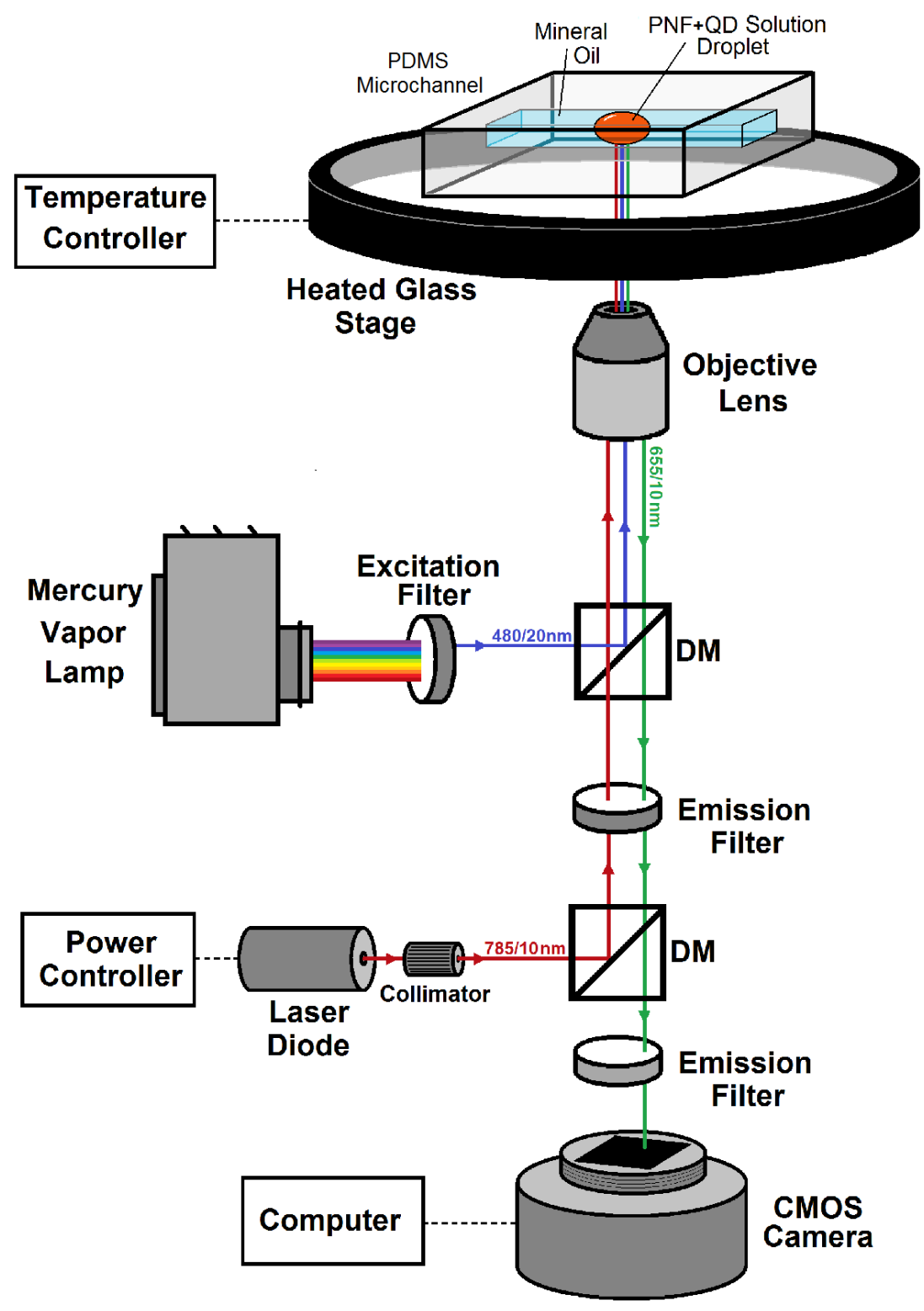

Figure 3.4 Primary Experimental Setup - Abstraction of the full experimental setup.

The primary optical experimental setup or this study consisted of an inverted microscope (Olympus IX-71 ) with a modified right-side attachment port. This 
modification allowed the separate and simultaneous illumination with excitation light sources for QD luminescence and photothermal heating. The primary excitation source for the QDs was a Mercury vapor arc lamp that was filtered to transmit light at a 450$500 \mathrm{~nm}$ wavelength band. This light was focused through the 10x magnification objective lens and onto the microscope stage. The secondary excitation source for the optical heating was a 785nm wavelength fiber optic laser (B\&W Tek). This laser has a linearly variable output power $0-450 \mathrm{~mW}(0-175 \mathrm{~mW}$ effective) and was narrowed through a collimator lens, a series of internal dichroic mirrors and filters, objective lens, and onto the microscope stage. Emission light was collected at $180^{\circ}$ from the excitation light path. This light was first filtered to wavelengths only between 610$657 \mathrm{~nm}$ and was then detected by the 1.3MP Color CMOS camera and collected via USB for processing.

The effective heating laser power $P_{\text {eff }}$ incident at the droplet was calculated to accommodate for the total transmission loses of the beam through the experimental setup. Light transmission values, $\mathbb{T}$, of the laser spectrum were obtained from the measured manufacture's data of each part passed through and compounded.

$$
\begin{aligned}
& \left.\left.\left.\left.\mathbb{T}\right|_{\text {collimator }} \cdot \underset{\text { lens }}{\left.\mathbb{T}\right|_{\text {secondary }}} \cdot \underset{\text { DM }}{\mathrm{T}}\right|_{\text {filter }} \cdot \underset{\text { fission }}{\mathrm{T}}\right|_{\text {primary }} \cdot \underset{\text { lens }}{\mathrm{T}}\right|_{\text {obj }} \cdot \mathbb{T} \mid \underset{\text { plate }}{\text { ITo }} \\
& \left.\cong \mathrm{T}\right|_{\text {total }} \\
& P_{\text {output }}^{\text {laser }} \cdot \mathrm{T}||_{\text {total }} \cong P_{\text {eff }}
\end{aligned}
$$




\subsubsection{Temperature-Fluorescence Calibration}

All PNF solutions in these series of experiments were prepared from one of three different commercially available gold nanoparticle aqueous suspensions: an 6011nm silica core GNS solution (Nanospectra), a 10-38nm GNR solution (Nanopartz), and a 10-102nm GNR solution (Nanopartz). Each gold nanoparticle solution was additionally prepared with an aqueous buffered solution of $\mathrm{CdSe}, \mathrm{ZnS}$ coated quantum dots (Invitrogen, Qdot655) with 655nm emission (Figure 3.1b). The spectra in Figure 3.5 show the absorbance (also called optical density) of each nanoparticle solutions measured with a vis-IR spectrometer. TEM images of the related GNR and GNS solutions are also given in the inset. Figure $\mathbf{3 . 5}$ clearly shows a large difference in optical densities for both nanoparticle solutions. because the GNR has a significantly higher particle contreaction than the GNS solution; resulting in 2 orders of magnitude larger absorption cross-section at the LSP wavelength. It should be noted, that optical density is defined as the logarithmic ratio of transmittance. Suggesting, that because the measured quantities are for the same sample thickness, the undiluted GNR solution is effectively $1^{100}$ times more absorbent to light at LSP wavelength than the compared GNS solution in this case. 


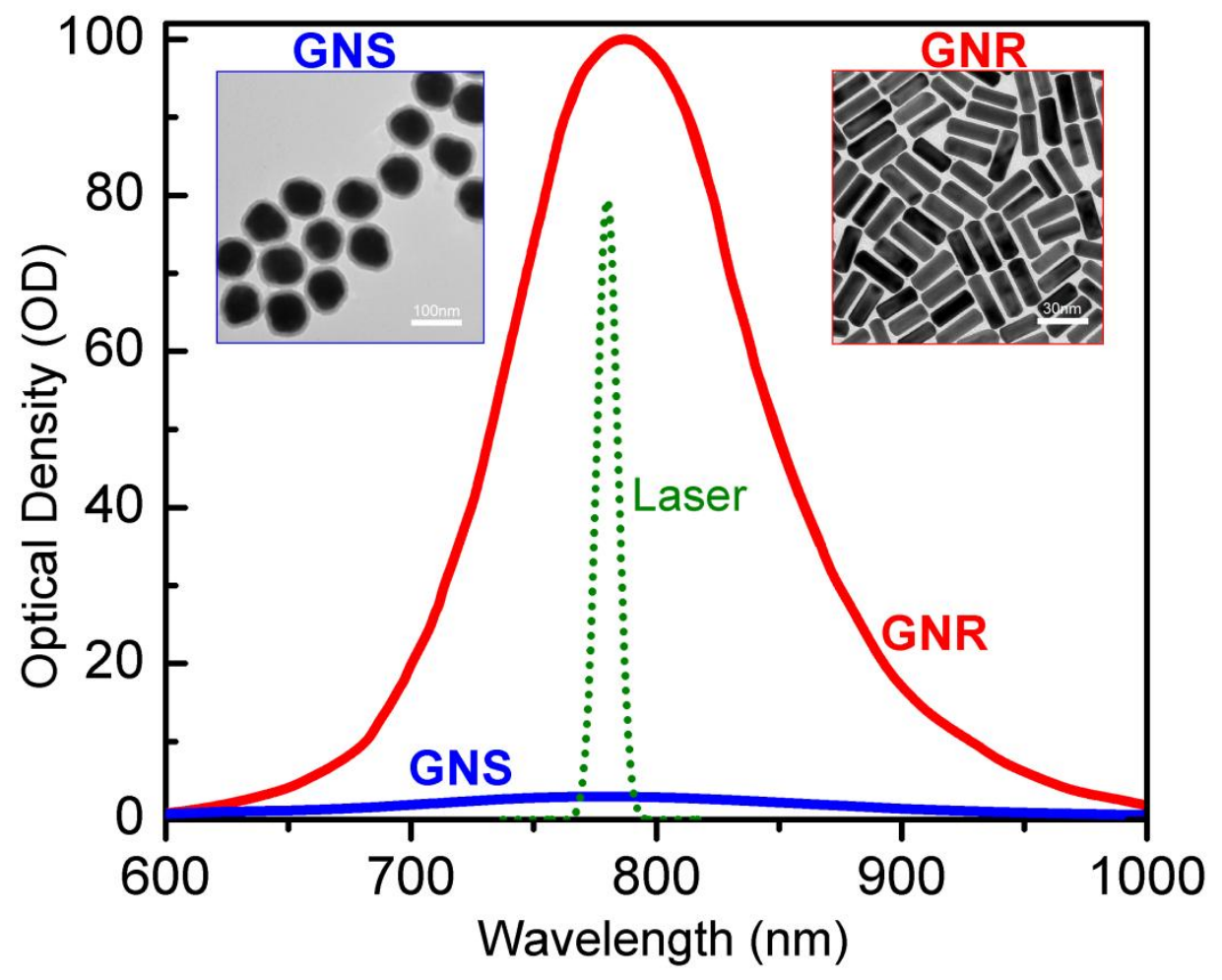

Figure 3.5 Nanoparticle Absorbance - Optical density spectrum for the undiluted GNS and GNR solutions with corresponding TEM images. TEM scale-bar: (GNS)100nm, (GNR)30nm.

Quantum dots were chosen over conventional fluorophores (i.e. Rhodamine B) for the purpose of optical thermometry for two main reasons. Quantum dot fluorescence emission intensity is known to be linearly proportional to temperature $[48,49,50]$, whereas most conventional fluorophores demonstrate an exponential or other complicated non-linear behavior [50,51]. This predictable behavior found in quantum dots is favorable as it greatly simplifies fluorescence-temperature correlations for optical thermometry. The second reason considered is that quantum dots are significantly more resistant to photobleaching than conventional fluorophores [48,50]; especially with regards to high power laser irradiation where this phenomenon can occur extremely quickly. This is an important consideration for over 
longer periods of laser cycling where the fluorescence emission of conventional fluorophores may eventually result in total photobleaching. Moreover, It can be noted that quantum dots possess a much higher quantum yield for fluorescence than that of conventional fluorophores, which is particularly beneficial in situations of small droplet volumes where emission signals may, otherwise, be too faint.

In order to prepare well-dispersed nanoparticle suspensions, they were first sonicated (Elma, Elmasonic P) for 30 minutes at $37 \mathrm{kHz}$ and $120 \mathrm{~W}$ power. Solutions were then placed in a beaker and mixed in several concentrations with a magnetic stirrer; borosilicate was also added as buffer to make a $\mathrm{pH}$ of 9 after the nanoparticle solutions were mixed with quantum dot solutions. All PNF+QD solutions were sonicated for 30 minutes at $37 \mathrm{kHz}$ and $120 \mathrm{~W}$ power prior to experiments. Figure 3.6 depicts a diagram of the experimental setup utilized for the initial temperaturefluorescence calibration. 


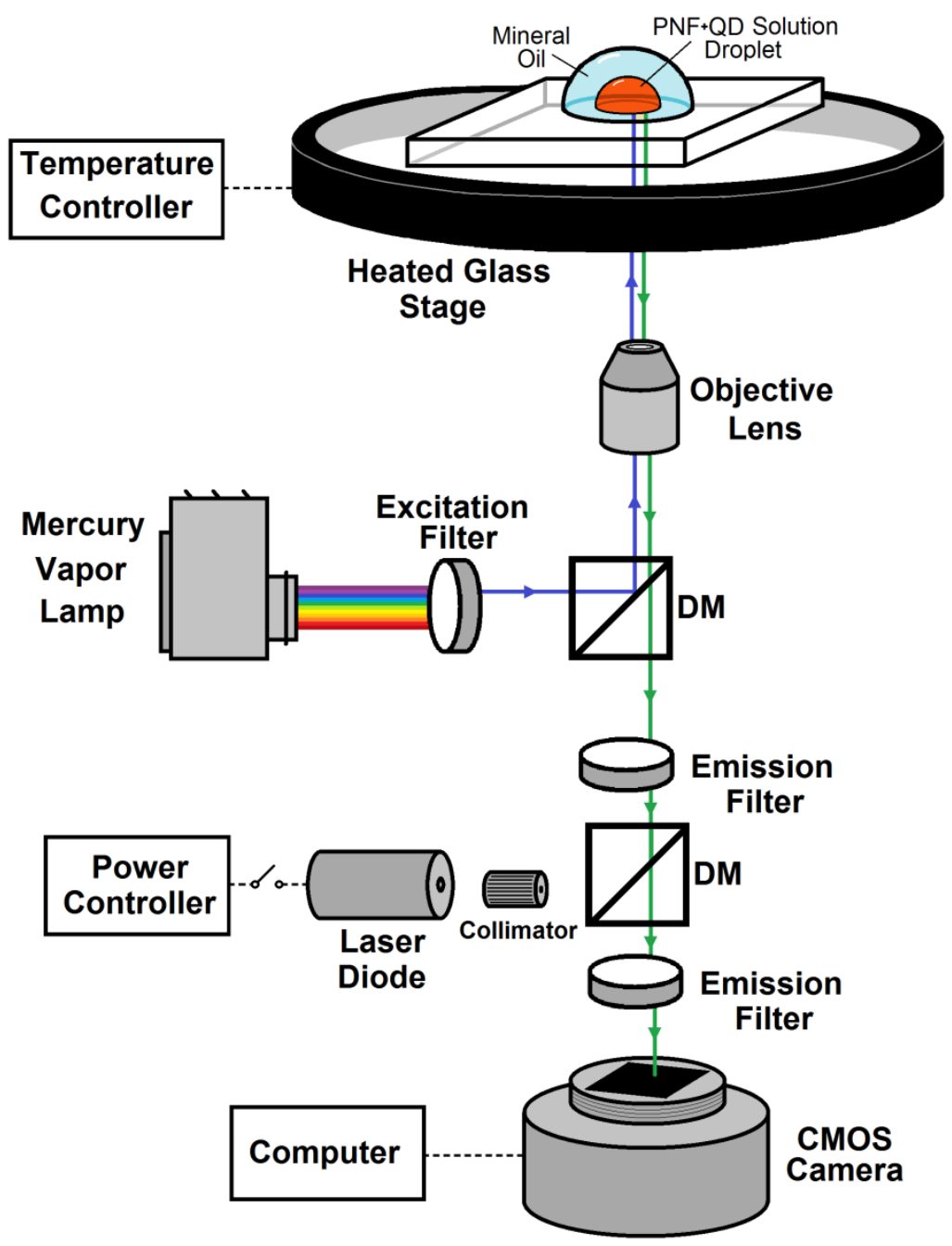

Figure 3.6 Temperature-Fluorescence Setup - Diagram of the temperaturefluorescence experimental setup.

A pipette (Fischer Scientific) was used to create individual hemispherical droplets of mineral oil USP on a PTFE-coated, standard microscope slide. The pipette was then inserted into the mineral oil USP droplet and used to suspend a $\sim 10-100 \mathrm{~nL}$ droplet of the PNF+QD solution. Next, the microscope slide was staged in an Indium Tin Oxide (ITO) glass heating plate with temperature controller (Bioscience Tools), which was affixed to the inverted microscope stage. The heater temperature was incrementally controlled from room temperature to $100^{\circ} \mathrm{C}$ with sufficient time to reach 
steady state. The fluorescence images of the suspended PNF+QD droplet were recorded via CMOS at each temperature increment. After a full temperature range of images were obtained, each image was then post-processed by measuring the average pixel brightness and normalizing relative to the initial temperature measurement at room temperature. This procedure was repeated 20 times for every PNF+QD solution to obtain the average values and uncertainty. This data was plotted and used to find and describe the linear relation of the fluorescence emission intensity to droplet temperature.

\subsubsection{Droplet Laser Heating}

The same PNF+QD solution preparation procedure was utilized in the laser heating experiment. A PDMS microchannel assay chip was first constructed with a Tjunction microchannel of $150 \mu \mathrm{m}$ depth and $400 \mu \mathrm{m}$ width. The PDMS surfaces were oxidized by air plasma in a plasma asher to fascilitate the hydrophillic interaction of the microchannel and fluid. The microchannels were first flushed through with isopropyl alcohol, dried, and then filled with mineral oil USP using a syringe pump. Next, an automated syringe pump (Guilfoyle Inc.) was utilized to control the flow rates of the mineral oil USP and PNF+QD solution through the T-junction; and a flow rate of $\sim 0.1 \mu \mathrm{L} / \mathrm{min}$ and $\sim 0.06 \mu \mathrm{L} / \mathrm{min}$ were chosen, respectively. By controlling the

flow of these two immiscible fluids, PNF+QD droplets can be generated with a vulume of $\sim 10-100 \mathrm{~nL}$ per $\sim 1 \sec [$ 52]. These PNF+QD droplets were pushed through into the microchannel as shown in Figure 3.7 and the flow was halted. 

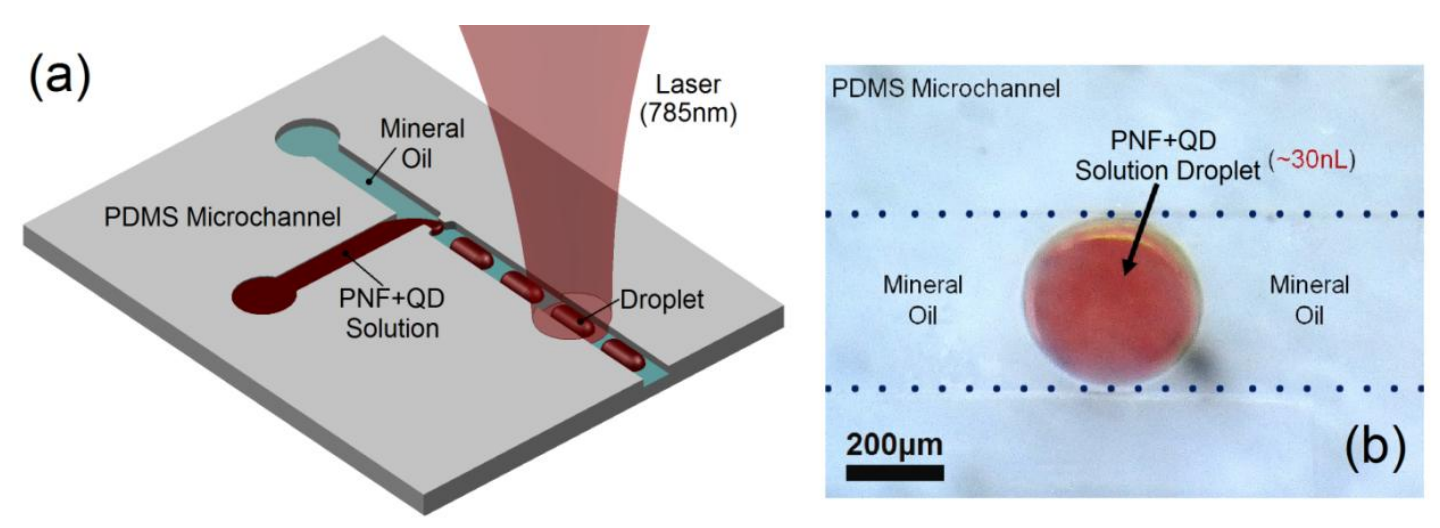

Figure 3.7 Droplet Laser Heating - (a) Microhchannel droplet laser heating schematic. (b)Microscope image of PNF+QD solution droplet in the microchannel.

The laser and Mercury vapor arc lamp light paths were aligned with the microdroplet and then slightly defocused to allow for better uniformity of incident light at the droplet surface. Next, the laser output power was manually modulated while the CMOS camera captured either images or video of the fluorescence emission at specific intervals of time. In the case of video, the CMOS camera was operated at the maximum frame rate of 107 frames per second over the duration of experimentation.

After a full range of laser output power images were obtained, each image was post-processed with imaging software (ImageJ) by measuring the average pixel brightness within the boundaries of the droplet. Next, these values were normalized relative to the room temperature fluorescence emission. The normalized data was compared with the data of the previously calibrated temperature-dependant fluorescence emission intensity versus temperature for each corresponding PNF+QD mixture, as well as with the laser manufacturer's measured data for power output $(\mathrm{mW})$ versus the controllable input current $(\mathrm{mA})$. By linking all three of these 
different relations, the temperature of the PNF droplet could then be correlated with the excitation laser output power density, simply calculated by, $P^{\prime \prime}=P_{\text {eff }} / A$. Here, $P_{\text {eff }}$ is the effective laser power output after transmission loses and $A$ is the area
out spot of the incident laser beam. It should be noted, that this is equation assumes a uniform distribution of intensity in the case of an de-focused incident beam as utilized in this work. 


\section{CHAPTER 4}

\section{FINDINGS}

\subsection{Simulation}

The results of the FEA simulation of the PNF droplet heating in the microchannel are shown in Figure 4.1. After the initial simulations to verify the viability of experimental pursuit, the simulations were rerun for theoretical matching.
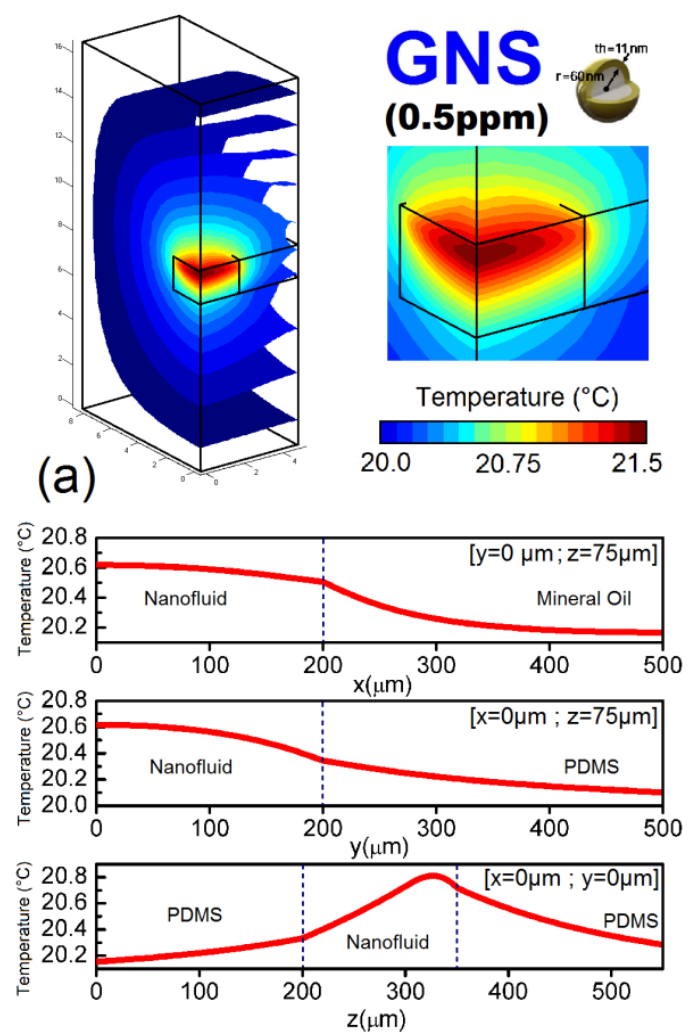
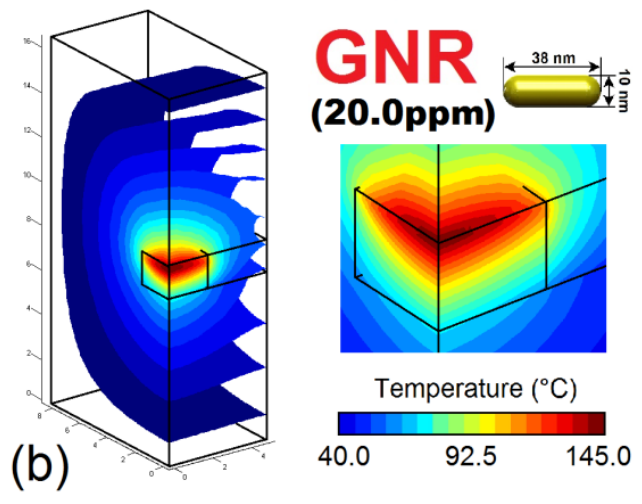

Temperature $\left({ }^{\circ} \mathrm{C}\right)$
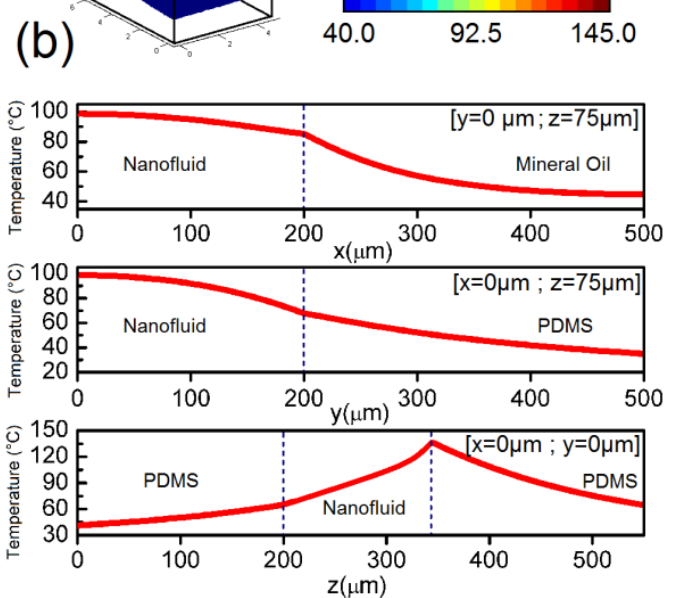

Figure 4.1 Simulation Result Matching - Simulated temperature steady-state distribution under $500 \mathrm{~W} / \mathrm{cm}^{2}$ for (a) $0.5 \mathrm{ppm}$ GNS concentration (b) 22.0ppm GNR concentration. Top to bottom: 3D isothermal plot, $\mathrm{x}$-axis temperature profile, $\mathrm{y}$-axis temperature profile, and $\mathrm{z}$-axis temperature along microchannel center. 
Figure 4.1 shows the simulation results for the corresponding experimental case conditions (discussed in section 4.2). It can be clearly seen that at $500 \mathrm{~W} / \mathrm{cm}^{2}$ laser power density, the GNS case is only capable of raising the average temperature of droplet by $\sim 1^{\circ} \mathrm{C}$ and GNR case by $\sim 70^{\circ} \mathrm{C}$. Both of these simulated average droplet temperatures fall within $90 \%$ of the measured average droplet temperature at that power density via Figure 4.4. Furthermore, the temperature distributions throughout the droplet are non-uniform. While some phase changing behavior was observed experimentally in this specific GNR case, there was absolutely no droplet bulk phase transition observed as would be implied by these simulation results. It is believed that the matching error and this non-uniformity could be accounted for with by consdering fluid convection within the physics of the COMSOL model.

The penetration depth of the excitation light serves a crucial role in the spatial distribution of the volumetric heat generation and consequent temperature responses of the plasmonic nanofluids. A clear characterization is necessary in understanding and verifying the heating behavior observed in this study. Figure 4.2 demonstrates the penetration depth, defined here as the depth within the PNF effective medium where the incident light reaches $\left[1-e^{-1}\right]$ of total extinction or $e^{-1}$ of incident intensity, as a function of the nanoparticle concentration. The combined right axis of this figure demonstrates the general regime of the temperature distributions throughout a microdroplet. The depth of the right-hand axis microchannel depictions was reduced from $150 \mu \mathrm{m}$ to $50 \mu \mathrm{m}$ to more clearly show the variation. 


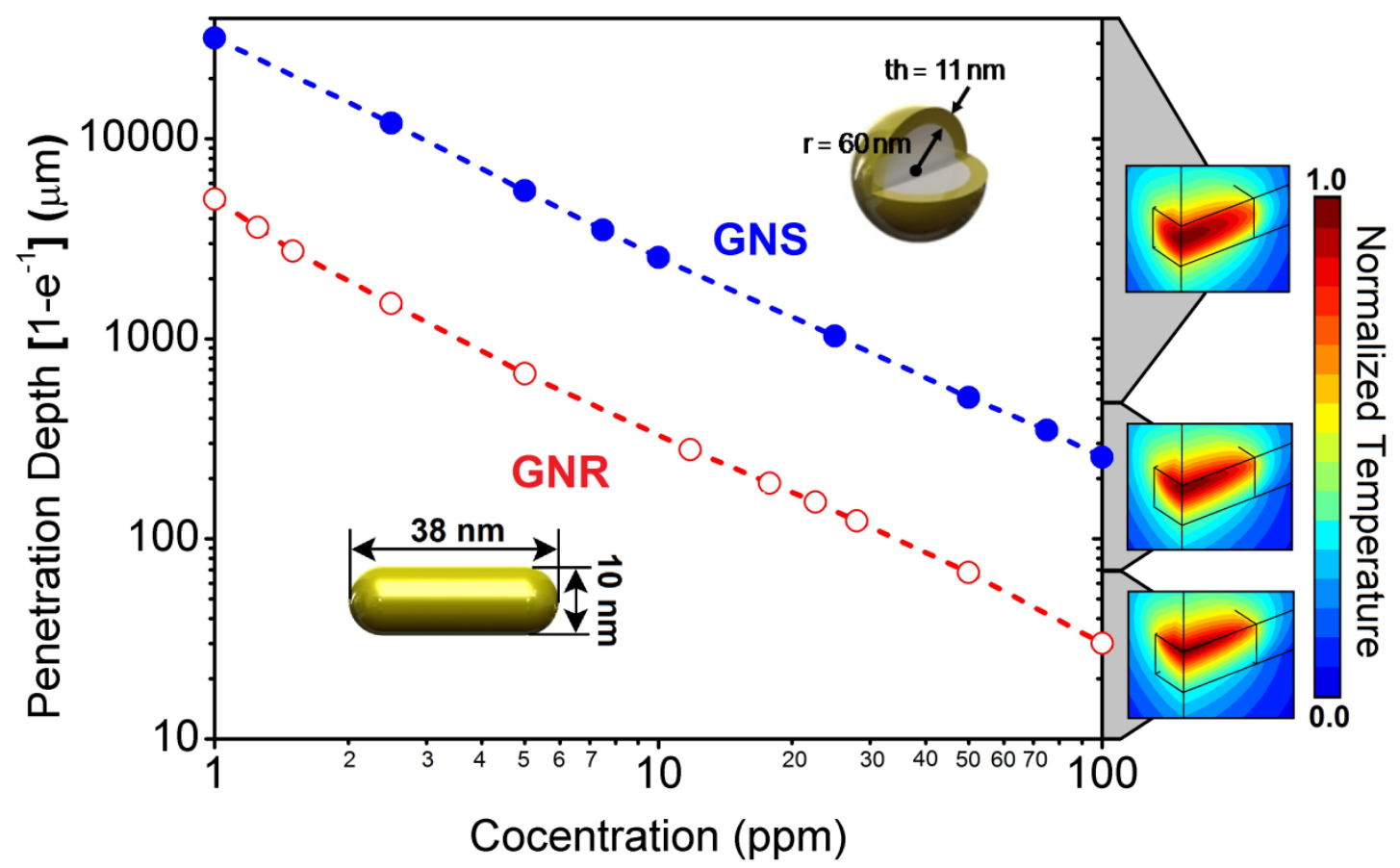

Figure 4.2 Penetration Depth - Calculated penetration depth of $785 \mathrm{~nm}$ laser spectrum for varying GNR and GNS concentrations. The right-hand axis depicts three generalized regimes of varying temperature distribution in a droplet of $50 \mu \mathrm{m}$ depth as a result of the corresponding light penetration depth for that case.

Although a uniform temperature distribution is desirable in our system during laser heating, it can be seen that there is the clear trend towards a significant concentration of heat generation and temperature increase within only the upper portion of the microdroplet. This tendency is of more immediate interest with regards to the GNR case, as the highest concentrations used during experimentation fall within this intermediate regime. With such a large temperature gradient present in the higher concentrations, the effects of local fluid natural convection would occur to be nonneglibile under these conditions. Although convection within the droplet was not simulated in the FEA model, it is likely that its effects may significantly aid to improve the temperature distribution for these mixture concentrations. 
From Figure 4.2, the light penetration depth for the GNS concentration used in experimentation $(\sim 1 \mathrm{ppm})$ is estimated to be $3 \mathrm{~cm}$; this is approximately 10 times larger than the equivalent GNR concentration, and nearly 200 times larger than the microdroplet depth. This is to say that most of the incident photon energy is not absorbed by the GNS mixture microdroplet at this concentration; supplemented by the very small temperature increase as shown resulting in the laser heating in Figure 4.2. Conversely, it is clear that the penetration depth for the maximum GNR concentration used in the experiment $(\sim 20 \mathrm{ppm})$ is estimated at $\sim 150 \mu \mathrm{m}$; nearly matching the total microchannel depth. This means that the predominant portion of available incident light is effectively absorbed by the GNR mixture microdroplet at this concentration.

\subsection{Experiment}

In order to ascertain accurate microdroplet temperature measurement, the temperature dependence of the fluorescence intensity change was calibrated. Figure 4.3 demonstrates fluorescent behavior observed for each PNF+QD mixture, GNS and GNR, while in a microdroplet suspended in an oil phase and heated through with the ITO heating plate. 


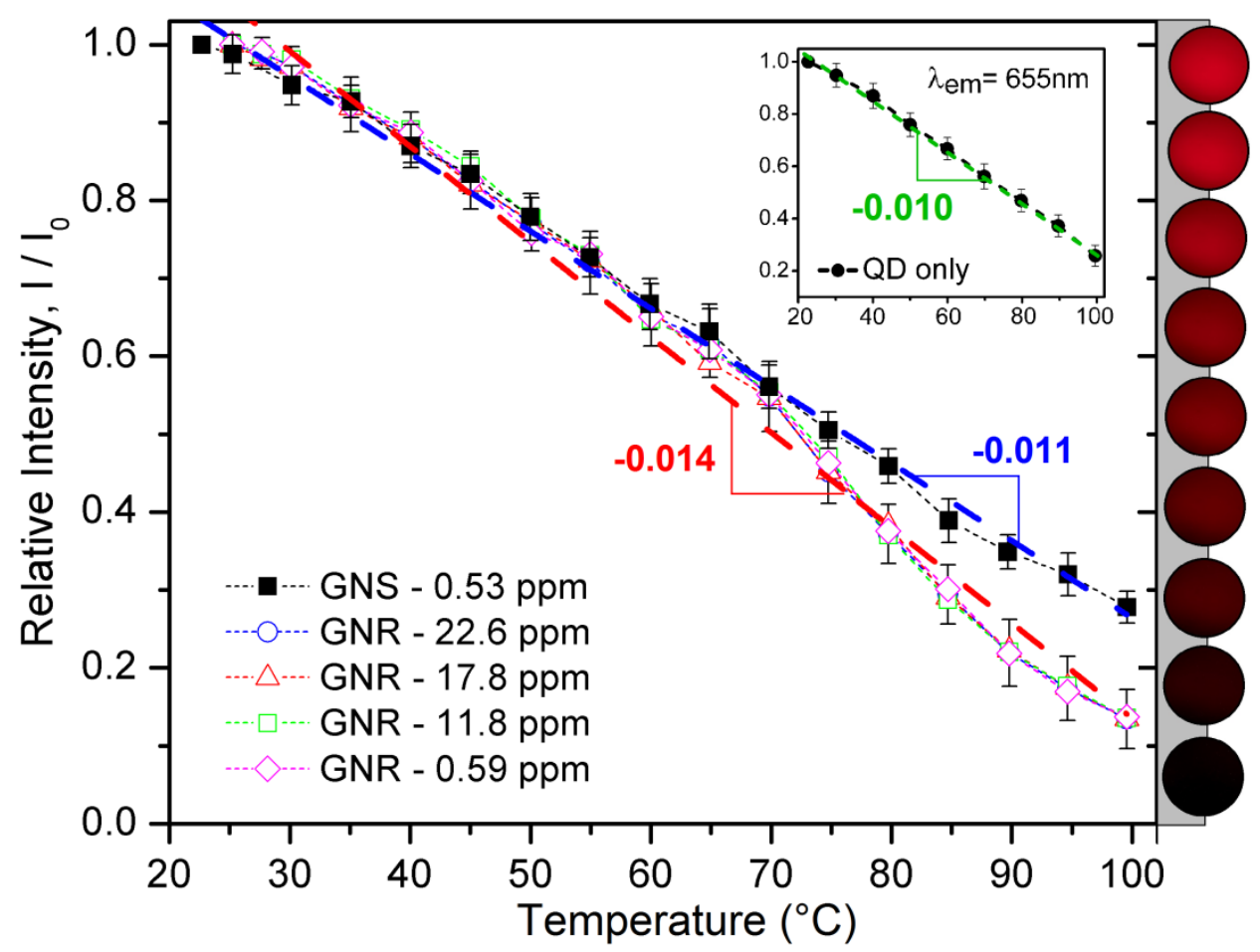

Figure 4.3 Temperature-Fluorescence Relation - Relative fluorescence intensity change of each PNF+QD solution droplet as a function of temperature. Right-hand axis depicts actual microscope images of the corresponding left-hand axis values.

For all PNF solutions, the fluorescence intensity linearly decreases as temperature increases; this being consistent with the trend of the QD solution without nanoparticles. The pure QD655 solution has a temperature-fluorescence relation of $-0.010 \mathrm{AU} /{ }^{\circ} \mathrm{C}$. The temperature-fluorescence relation of the GNS and GNR cases are $-0.011 A U /{ }^{\circ} \mathrm{C}$ and $-0.014 A U /{ }^{\circ} \mathrm{C}$, respectively. It is evident from Figure 4.3 that the nanoparticle concentrations prepared had very little or no effect on the overall trend through this range, as all cases for GNR-based solutions displayed near-identical behavior. However, the different nanoparticle structure suspension were observed to case an effect on the overall temperature-depdence, as both nanoparticle cases demonstrated an larger decrease than that of the pure QD solution. The reasons for these differences is not fully understood at this time, but it is most likely attributable to 
near-field electromagnetic interactions (i.e. Förster resonance energy transfer [53,54]) between the gold nanoparticles and the QDs within the solutions. Nevertheless, these slope values are consistent and characteristic only of which type of nanoparticle structures are mixed; thus, further showing the behavior is independent of the concentration of that mixture within the presented cases.

With the fluorescence emission-temperature relationship known, real-time correlation between the laser power and fluorescence emission can be measured. The laser heating measurements were repeated for each nanoparticle structure and mixture concentration in a microdroplet and microchannel as seen in Figure 4.4. All fluorescence data collected for this experiment was obtained for microdroplets with volumes equal to approximately $30 \pm 20 \mathrm{~nL}$, or $160 \pm 60 \mu \mathrm{m}$ in diameter. 


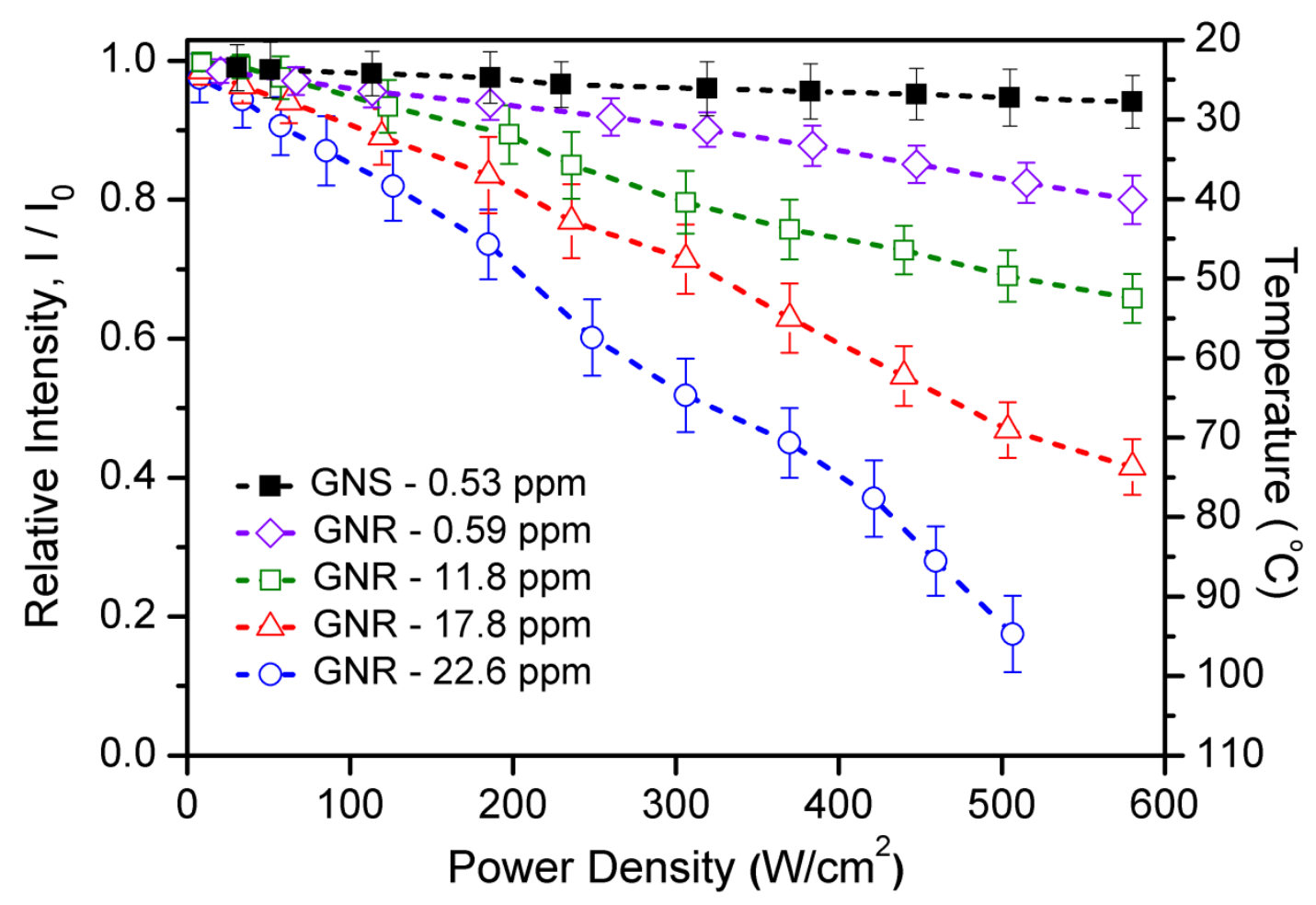

Figure 4.4 Plasmonic Laser Heating - Relative fluorescence intensity change for each PNF+QD solution droplet in microchannel as a function of laser power density.

Right-hand axis correlates relative intensity to inferred temperature.

It was found that the highest available concentration $(\sim 0.53 \mathrm{ppm})$ of the GNSbased mixture was only able to reach $\sim 26^{\circ} \mathrm{C}$ in droplet in the microchannel, or an approximate $5^{\circ} \mathrm{C}$ increase. Three GNR-based mixtures $(0.59,11.8$, and 17.8$)$ were capable of reaching $\sim 40,52$, and $75^{\circ} \mathrm{C}$, while the highest concentration (22.6ppm) is capable of reaching a temperature of $\sim 100^{\circ} \mathrm{C}$ within droplet. It should be noted that the 22.6ppm GNR mixture is capable of heating the droplet further Although the lowest concentration GNR mixture is nearly the same as that of the GNS mixture, its heating capability is $225 \%$ more effective, able to achieve a $\sim 10^{\circ} \mathrm{C}$ higher temperature increase. This was to be expected, as the utilized GNS solution had a maximum optical density of $\sim 4 \mathrm{AU}$ and the GNR solution had $\sim 100 \mathrm{AU}$ at $780 \mathrm{~nm}$ wavelengths.. 
This strongly suggests that GNR structures would serve optimally for smaller microdroplets that have far fewer nanoparticles due to its superior absorption efficiency.

In order to clearly verify that the effects of this droplet heating is predominantly attributable to plasmonic heating, Figure 4.5 depicts two dissimilar GNR structures with different LSP absorption peaks.

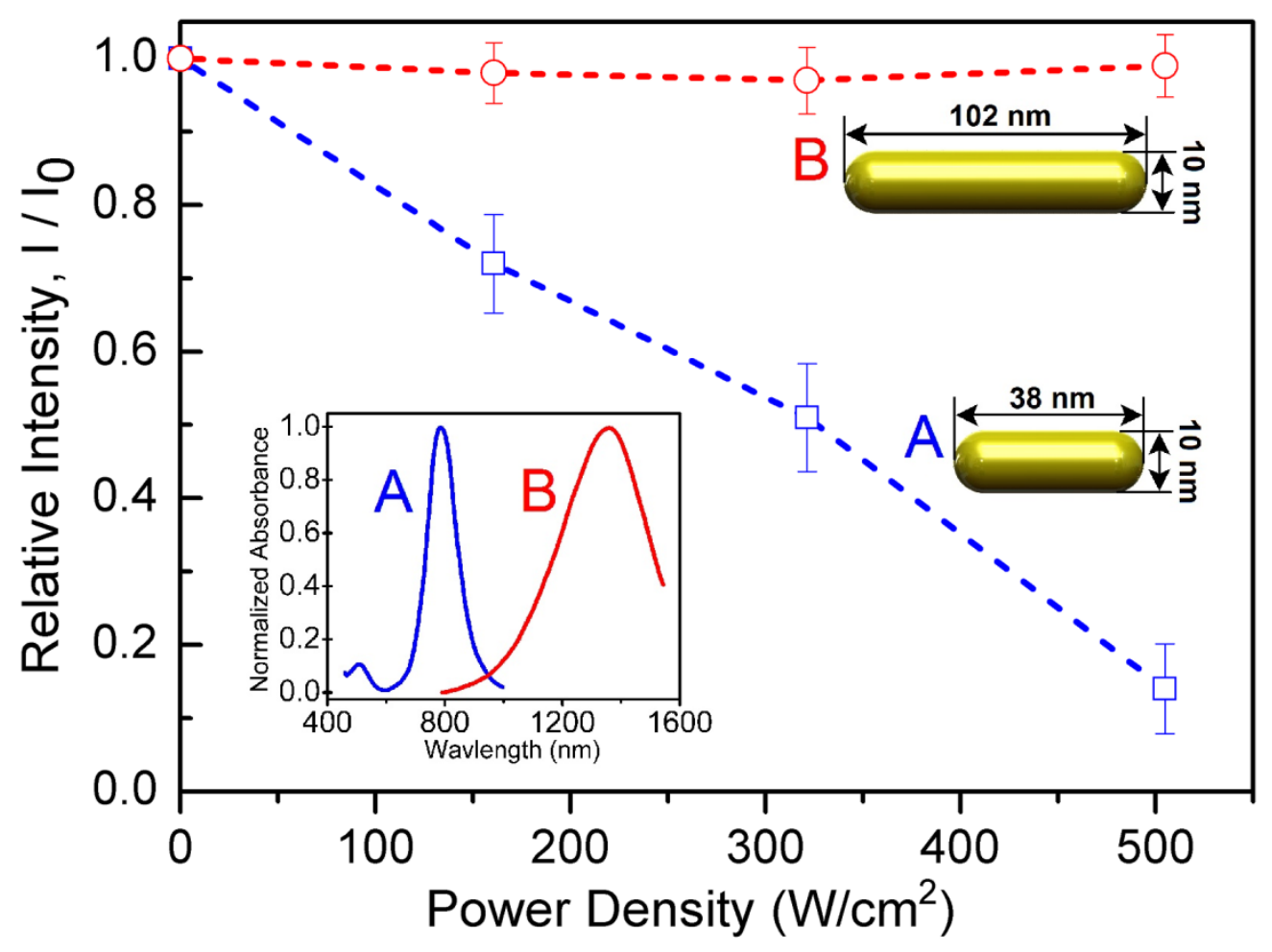

Figure 4.5 Plasmonic Heating Verification - Relative fluorescence intensity change versus laser power density for two GNR structures of different LSP wavelengths. Both

GNR cases prepared in equal concentration solution droplets with QDs and laser heated in microchannel.

Both GNR structures A and B were irradiated with the same laser spectrum and power density range, as well as the same approximate solution concentration and microdoplet size. As shown in the inset, GNR structure B exhibits an absorption peak 
at $\sim 1400 \mathrm{~nm}$; thus will have very little or no fluorescence intensity change when irradiated with the $785 \mathrm{~nm}$ laser. On the other hand, GNR structure A with a $\sim 780 \mathrm{~m}$ absorption peak, has a very clear fluorescence decrease under the same irradiation. It should be noted that all of the solutions used in this investigation were aqueous-based, however precaution was taken in the case of GNR structure B with regards to the strong absorption of light $(\mathrm{OD} \gg 1)$ that $\mathrm{H}_{2} \mathrm{O}$ exhibits in $\sim 1400 \mathrm{~nm}$ IR-spectrum. This spectral overlap would catastrophically interfere with accurate measurement of the LSPR behavior, so GNR structure $B$ was prepared in a deuterium oxide $\left(\mathrm{D}_{2} \mathrm{O}\right)$ solution as it does not strongly absorb light in the $1400 \mathrm{~nm}$ range $(\mathrm{OD} \cong 0.2)$, but still behaves chemically similar to $\mathrm{H}_{2} \mathrm{O}$.

Accurately determining the transient effects of the PNF droplet heating serves an imperative role in weighing its effectiveness in time-sensitive applications. As mentioned, PCR offers as a direct example of the very wide range of applications which could decidedly benefit from plasmonic heating integrated microfluidics. In order to achieve a full plasmonic photothermal PCR, a fast, precise, and repeatable thermal cycling behavior is desired to achieve shorter processing times. Figure 4.6 demonstrates the rapid and large temperature changes of the droplet during laser heating for a 22.6ppm GNR+QD microdroplet in a microchannel. The droplet was $\sim 50 \mathrm{~nL}$ in volume and the microchannel was $150 \mu \mathrm{m}$ in depth. 


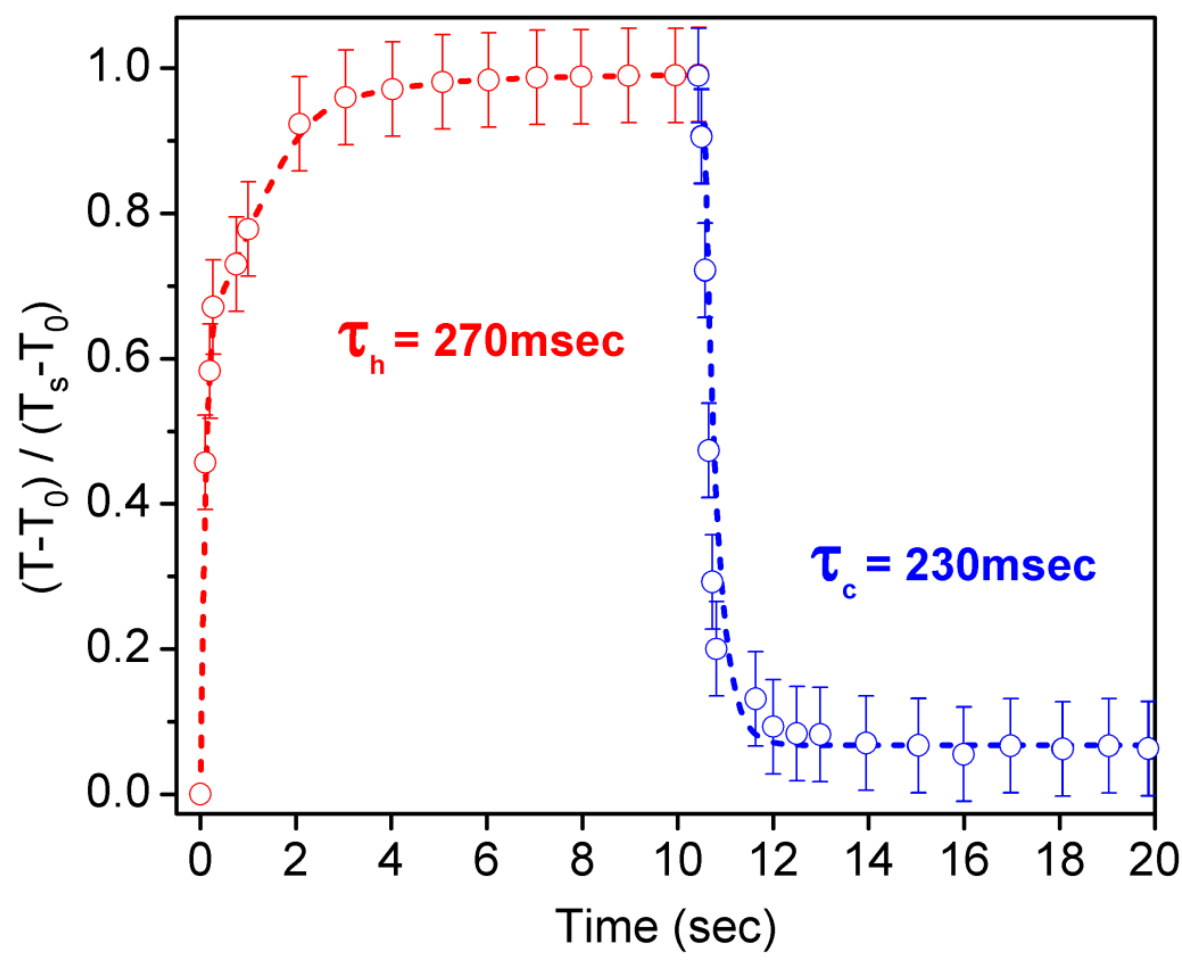

Figure 4.6 Transient Heating Response - Relative temperature change of GNR+QD solution droplet in microchannel versus time. 1 full thermal response cycle was performed over a 20 second interval. Average thermal time constant of heating was calculated to be $270 \mathrm{msec}$ and thermal time constant of cooling was $230 \mathrm{msec}$.

The laser was switched on (from 0 to $500 \mathrm{Wcm}^{-2}$ ) and then abruptly switched off during fluorescence measurement. The thermal time constant for heating is approximately $270 \mathrm{msec}$ and time constant for cooling is approximately $230 \mathrm{msec}$. This fast cooling is most likely attributed to higher conduction heat loses to the surrounding medium due to the large surface to volume ratio of the microdroplet. The rapid heating and cooling of this particular PNF solution was able to attain a $\sim 45^{\circ} \mathrm{C}$ change in $\sim 250 \mathrm{mec}$ at maximum rate or very roughly, $>25^{\circ} \mathrm{C} / \mathrm{sec}$ averaged over the full process. This is significant improvement compared to many of the best conventional PCR heating systems commercially available with a maximum rate of $\sim 10^{\circ} \mathrm{C} / \mathrm{sec}$. It should be noted that only the first step in PCR would require full thermal response on the 
PNF droplet; the other intermittent steps require lower temperatures and thus could be reached much faster. Additionally, it should be noted that the transient response times of this process could be potentially further improved by increasing the PNF concentration still higher.

Furthermore, Figure 4.7, demonstrates that this same heating and cooling process in the microdroplet is fully repeatable and consistent over many cycles for the GNR solution.

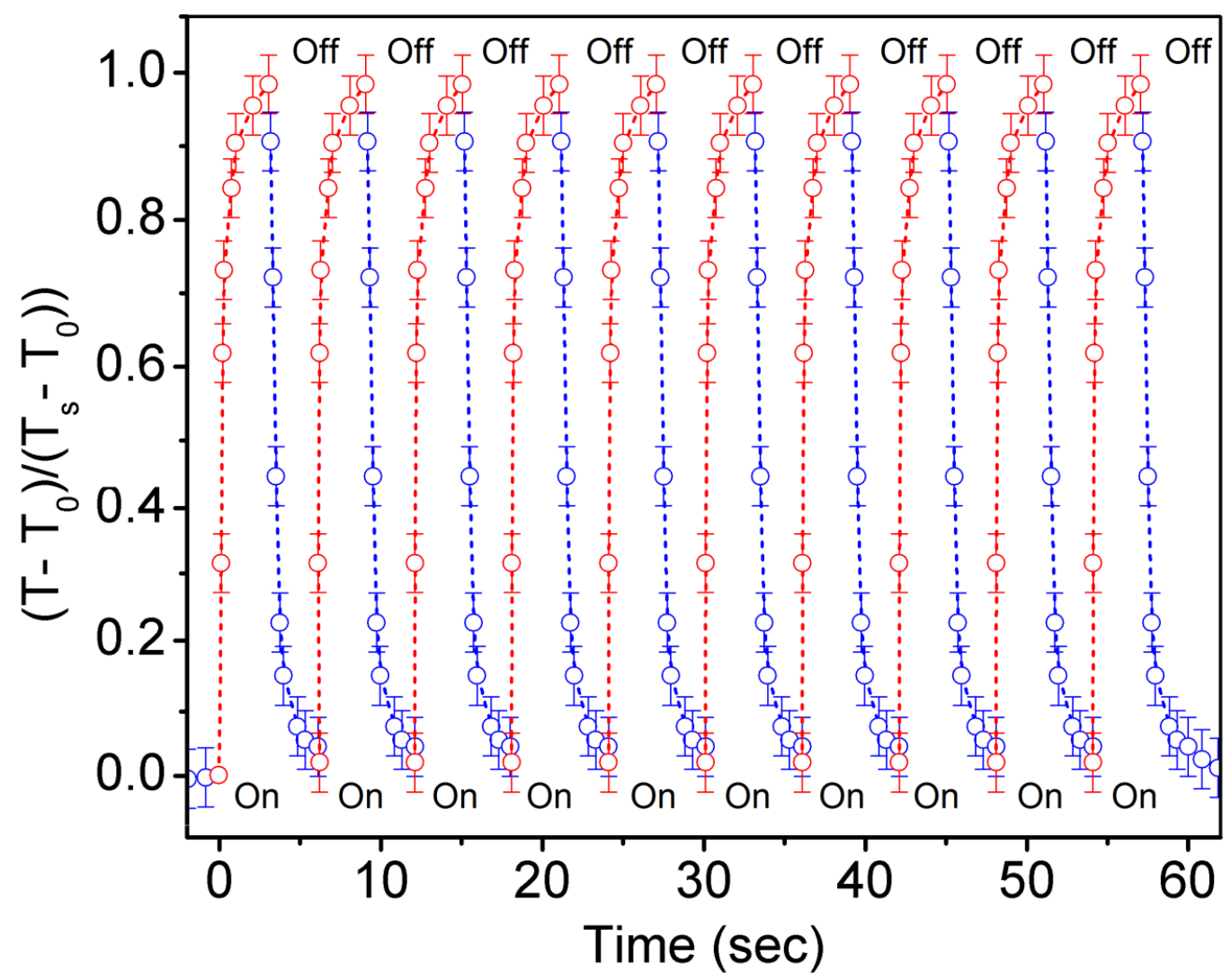

Figure 4.7 Transient Thermal Cycling - Relative temperature change of GNS+QD droplet in microchannel versus time. 10 full thermal response cycles were repeated over a 60 second with negligible fluorescence signal degradation. 
As a common PCR procedure may consists of 20-40 main cycles, relative long term repeatability is an imperative quality of this system. Figure 4.7 is a temporal continuation of Figure 4.6, showing the same procedure continuing over a total of 10 cycles. A common hot-start PCR requires 6 steps: initialization $\left(\sim 98^{\circ} \mathrm{C}\right)$, denaturation $\left(\sim 95^{\circ} \mathrm{C}\right)$, annealing $\left(50-65^{\circ} \mathrm{C}\right)$, elongation $\left(75-80^{\circ} \mathrm{C}\right)$, final extension $(70$ $\left.74^{\circ} \mathrm{C}\right)$, and final hold $\left(\sim 20^{\circ} \mathrm{C}\right)[4]$. While the figure above demonstrate cycling between full thermal responses, this exhibits a worst-case-scenario time range in a complete plasmonic PCR; the lower temperature ranges of the intermittent steps require shorter response times to reach and thus would greatly reduce full procedure times.

One point worthwhile to note is that that localized boiling and vapor generation was not readily observed during droplet laser heating in the microchannel experiment. This is believed to be as a result of the "confinement effect" [54]; a behavior observed by an aqueous liquid phase subsisting as a liquid phase at pressures and temperatures that would otherwise induce bulk cavitation and evaporation when confined between the gap of two microsclae non-permeable plates. However, localized boiling of the solution around GNR (but not GNS) agglomerations were readily observed in improperly homogenized dispersions during laser heating of droplets in the non-microchannel confined figuration outlined in section 3.2.2. These events often additionally resulted in intensely prominent effects of micro-thermopheresis when the excitation power reached a critical level. These effects would commonly cause fluid flows, voids within the PNF, and the eventual collapse of the well-formed water-oil phase boundary. To this end, great care was and must be taken to properly prepare the 
PNF solutions and control the extremely sensitive heating effects caused by the excitation source.

Also noteworthy, are the effects of heating in larger droplets with volumes $\leq 500 \mathrm{~nL}$ with nanoparticle concentrations $\geq 1 \mathrm{ppm}$, which exhibited a very noticeable convection column centered about the laser focal point within the droplet. This behavior was more commonly observed in the GNS solution for insufficiently small microdroplets and very little fluorescence change was detectable, despite obvious effects of temperature change. This is believed to be because of only a slight temperature difference existing due to the much lower absorption efficiency of the GNS and a drowning out of the fluorescence signal from the mixing of the un-heated surrounding PNF medium. 


\section{CHAPTER 5}

\section{CONCLUSION}

\section{1}

This work presented the creation of several multi-stepped computational models aimed to theoretically verify and characterize the laser-assisted heating behavior of GNS and GNR plasmonic nanofluid droplets in a microchannel. The absorption and scattering properties of the GNS structures for varying concentrations were determined by the Mie scattering equations, whereas the solutions to the BEM were utilized for the GNS structures. A Monte Carlo algorithm simulation was used to predict the spectral absorbance behavior for the GNS structures by the tracing photonnanoparticle interaction paths. The spectral absorbance was used to calculate the volumetric heat generation of the PNF for a given laser power and this was imposed on an 3-dimensional FEA model in COMSOL 3.5a used to solve for temperatures. Computed temperature solutions for low concentration mixtures were found to be generally agreeable with experimental results, however did demonstrate clear discrepancy - most likely attributable to a lack of consideration given to fluid convection in the FEA model. GNS concentrations $\geq 3000 \mathrm{ppm}$ and GNR concentrations $\geq 7 \mathrm{ppm}$ were found to achieve maximum temperatures $\geq 100^{\circ} \mathrm{C}$ in a droplet volume of $\sim 30 \mathrm{~nL}$. Overall, these models were found sufficiently versatile and accurate enough towards suggesting the possibility of successful experimental plasmonic heating in a microchannel. 
Experimentally, this work observed the fluorescence-temperature relation of GNR and GNS structures mixed with QDs for achieving real-time, accurate fluorescence-mediated thermometry. The effects of nanoparticle structure and concentration on the total temperature increase were examined for laser heating of microdroplets in a microchannel. GNR PNFs for concentrations $\leq 22.6 \mathrm{ppm}$ were found to be sufficient in achieving a full range of steady-state, sub-boiling temperatures. Further, the transient behavior during laser heating for these GNR concentrations were examined; thermal time constants were found to be in the order of $200 \mathrm{msec}$ with an average temperature rate $\cong 25^{\circ} \mathrm{C} / \mathrm{sec}$ and maximum measured temperature rate $\cong 180^{\circ} \mathrm{C} / \mathrm{sec}$. This transient temperature behavior was observed to be repeatable over numerous continuous cycles for several minutes with little or no degradation.

The heating behavior of these GNR mixtures was verified to be truly as a result of plasmonic heating, by repeating the same laser heating experiment conditions with a GNR structure of different LSPR wavelength. For this control experiment, no temperature increases were observed.

The results obtained in this work viably demonstrate the superb potential of plasmonic heating integrated microfluidic systems. Countless existing LOC applications, such as the Polymerase Chain Reaction, could greatly benefit in enhanced throughput and efficiency from the fast and accurate optical heating methods presented in this work. 


\section{APPENDICES}

\subsubsection{Mie Scattering Code}

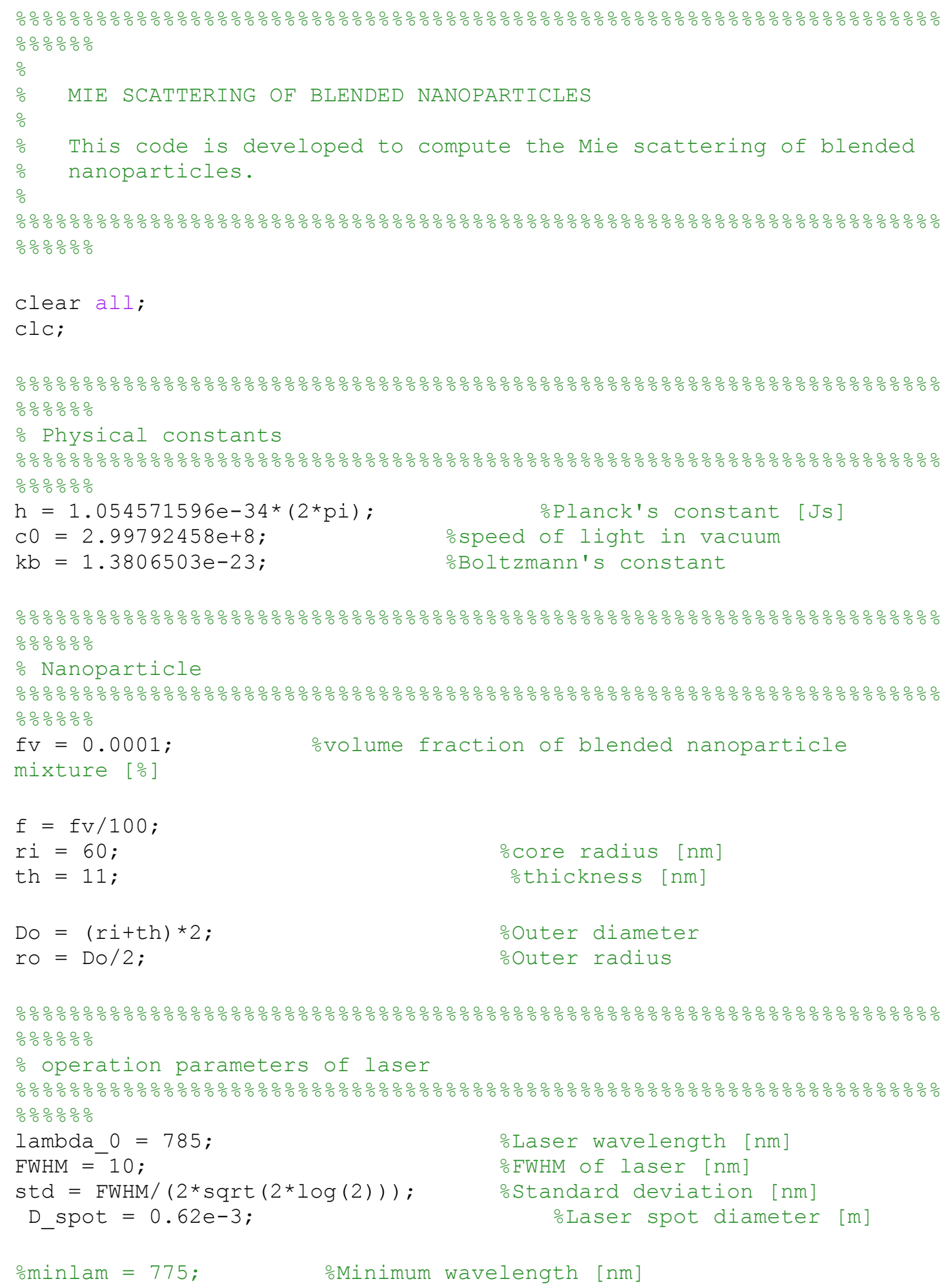




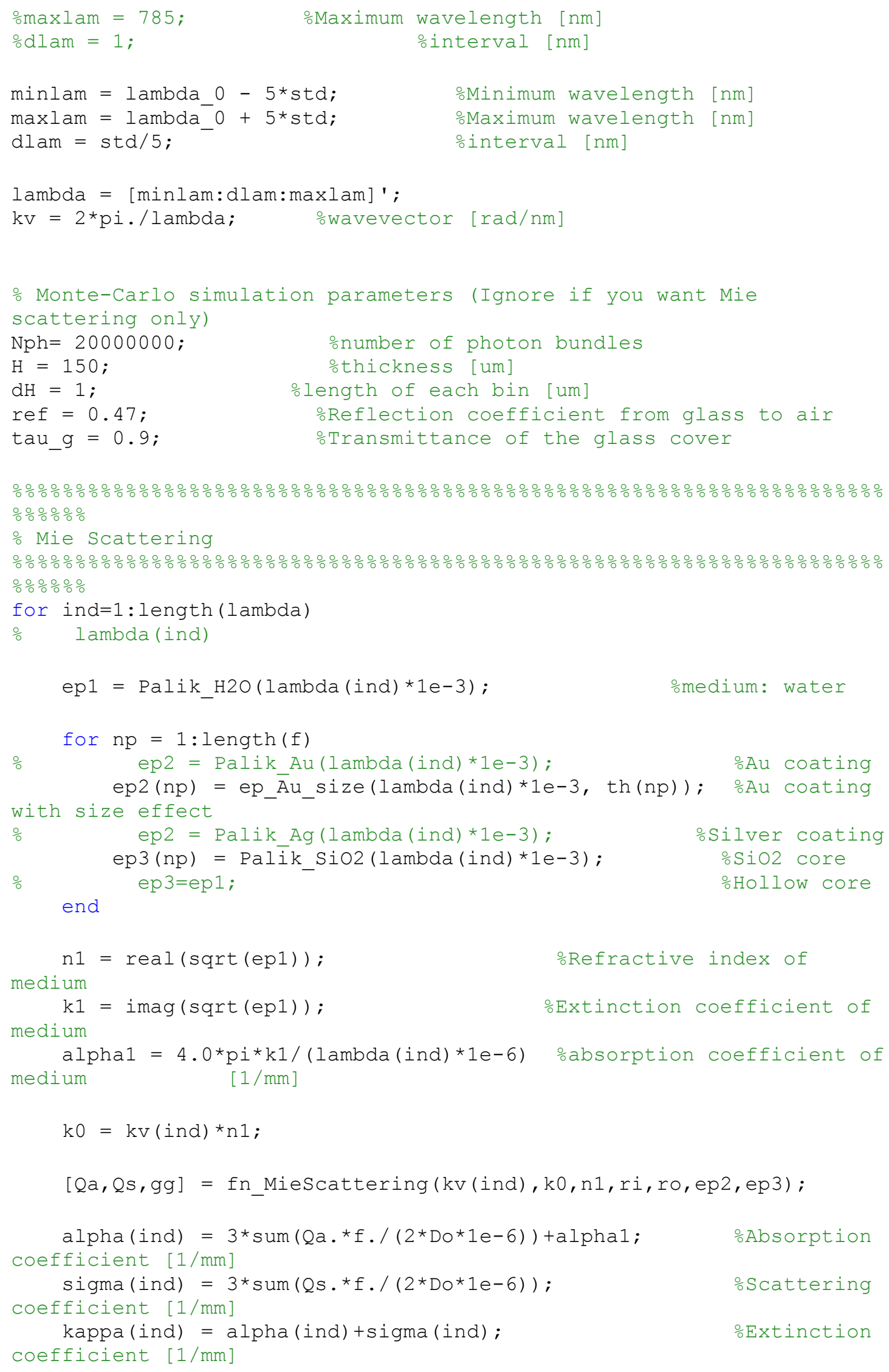




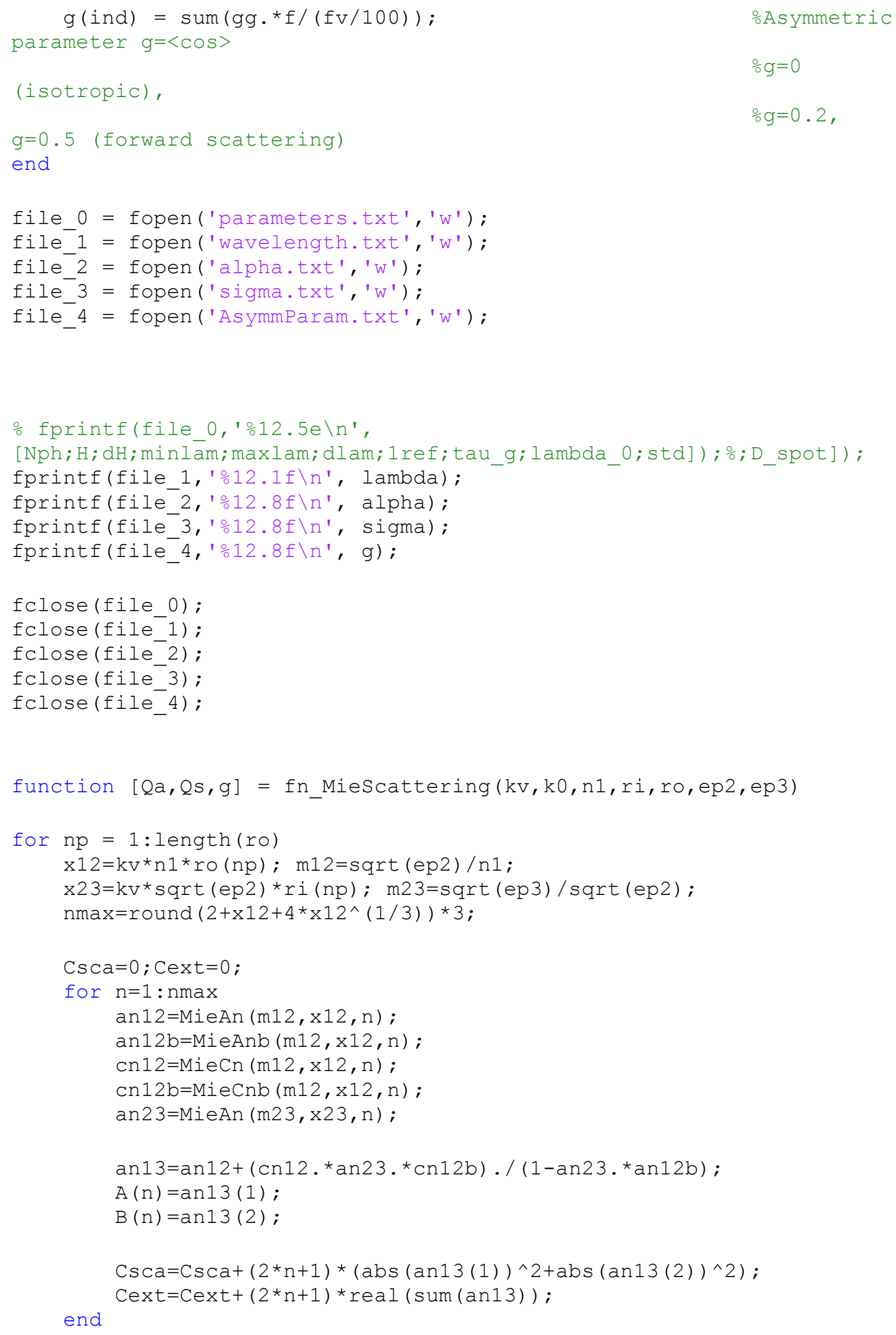




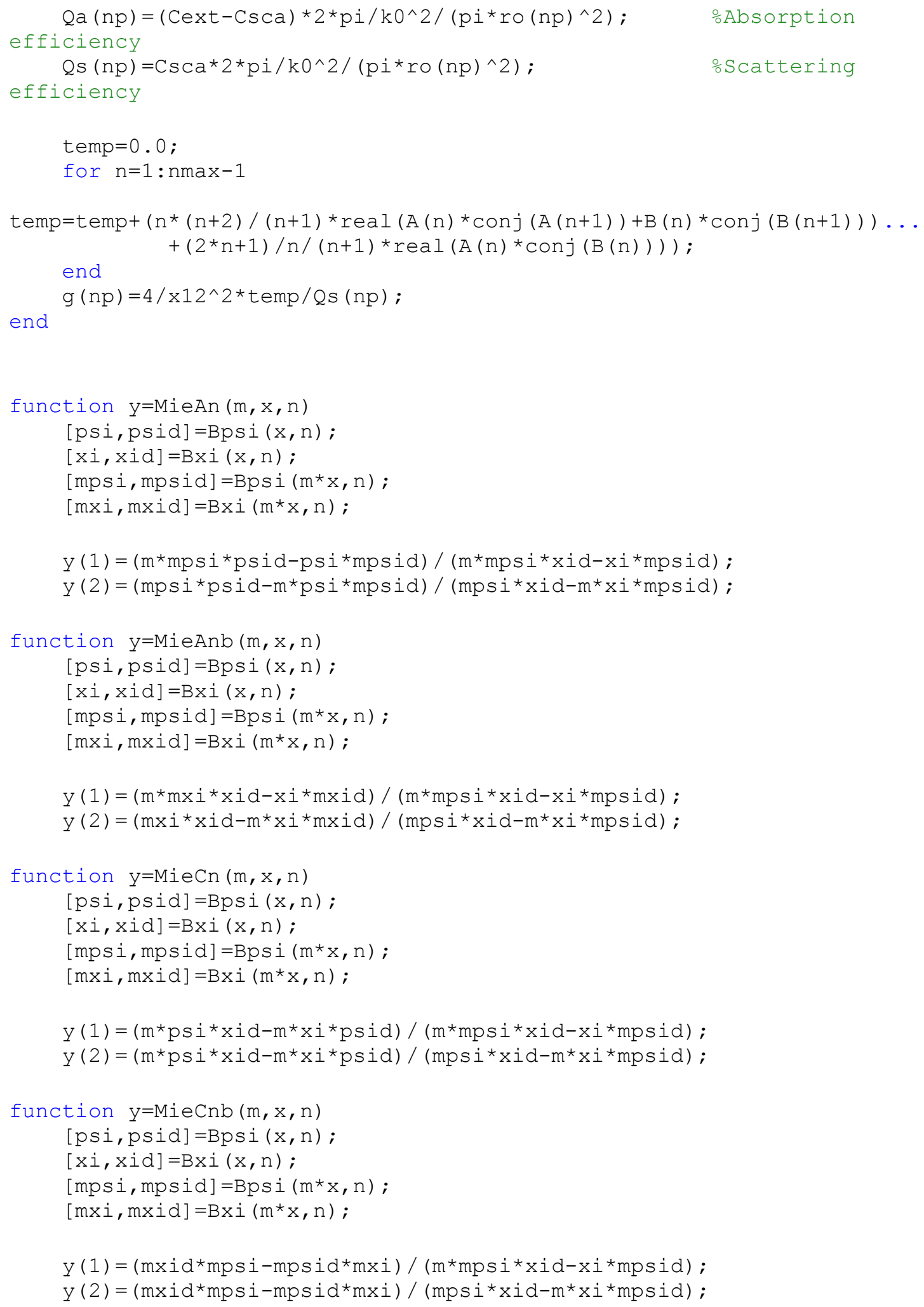




\subsubsection{Monte Carlo Simulation}

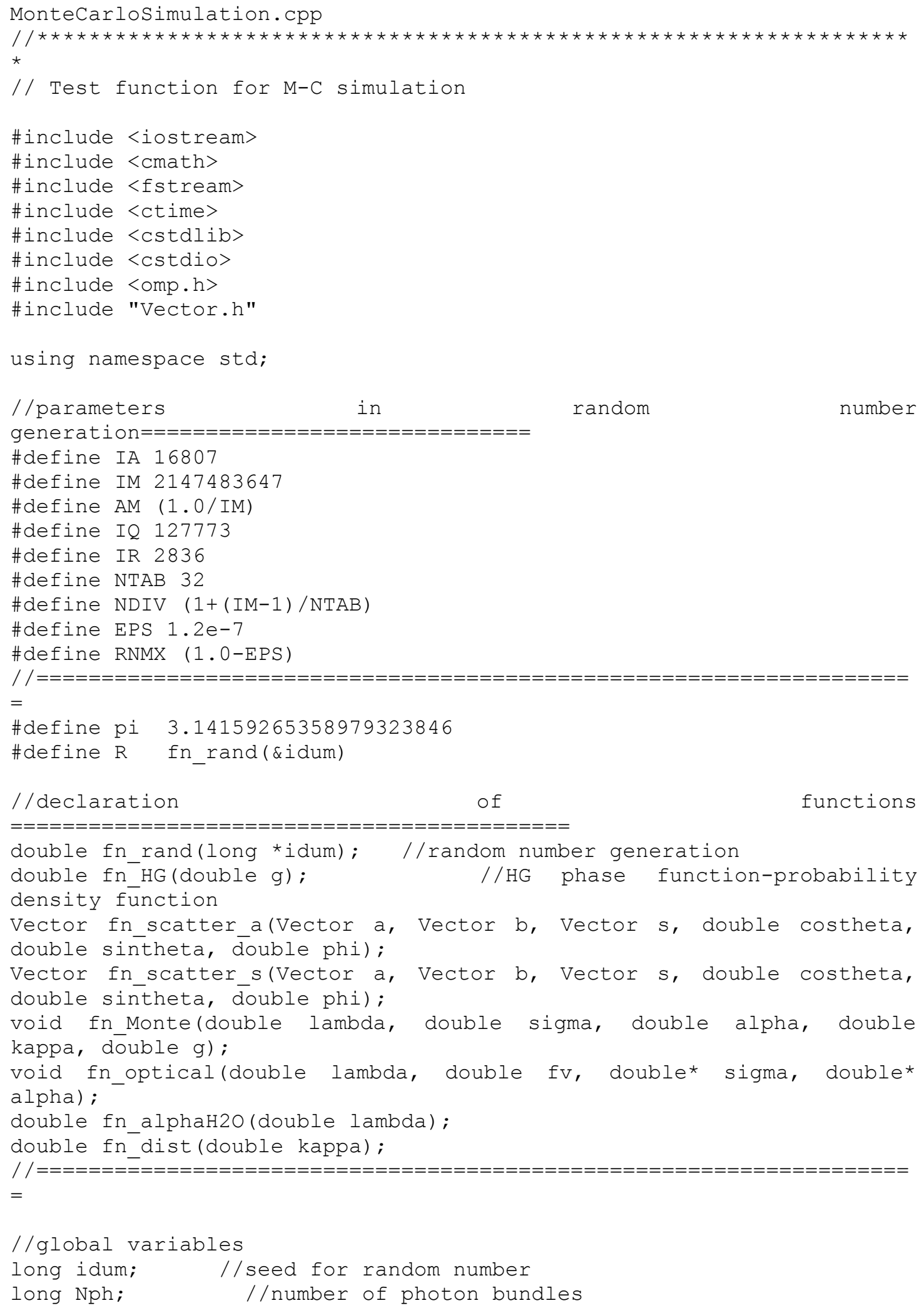




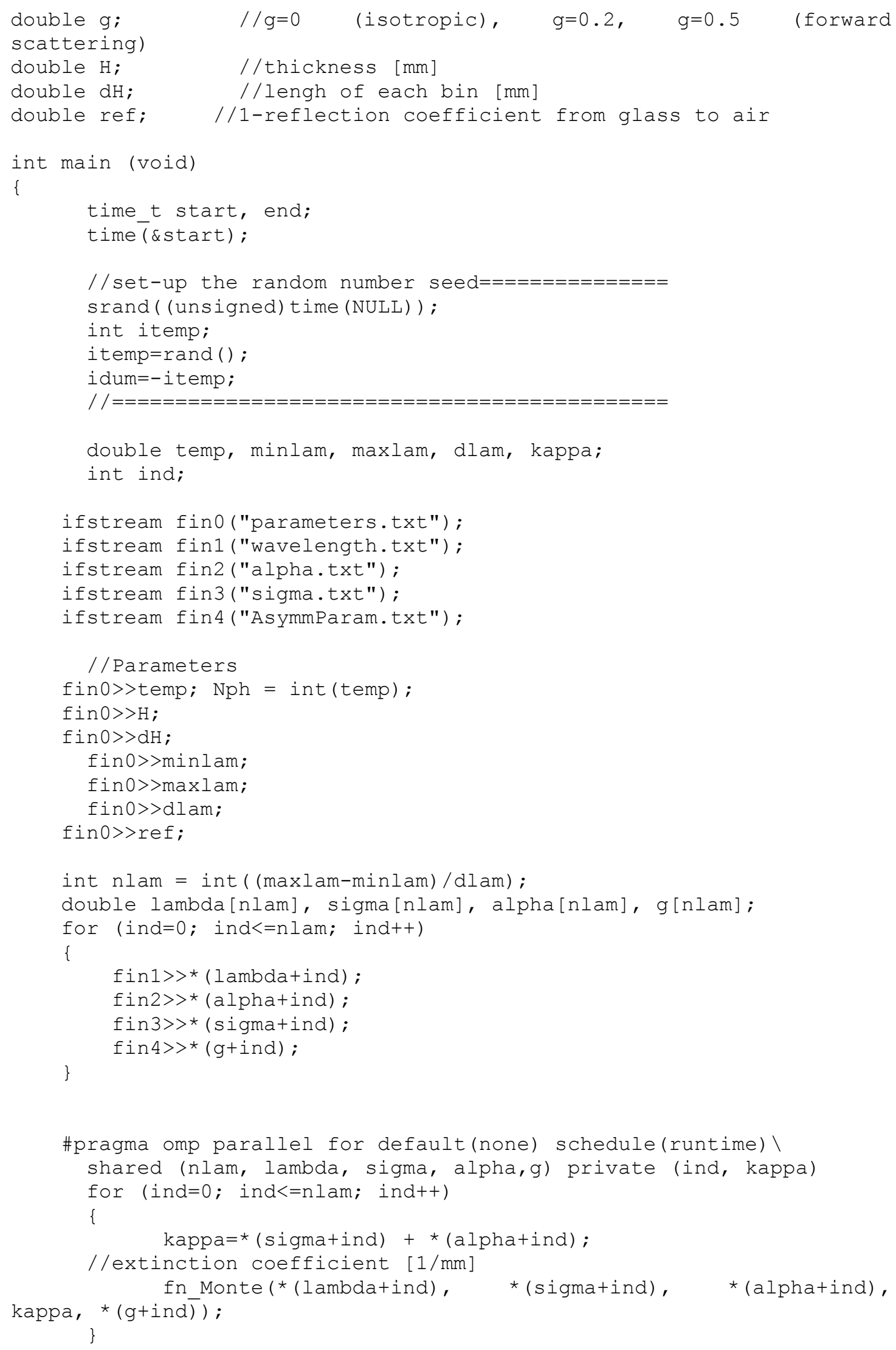




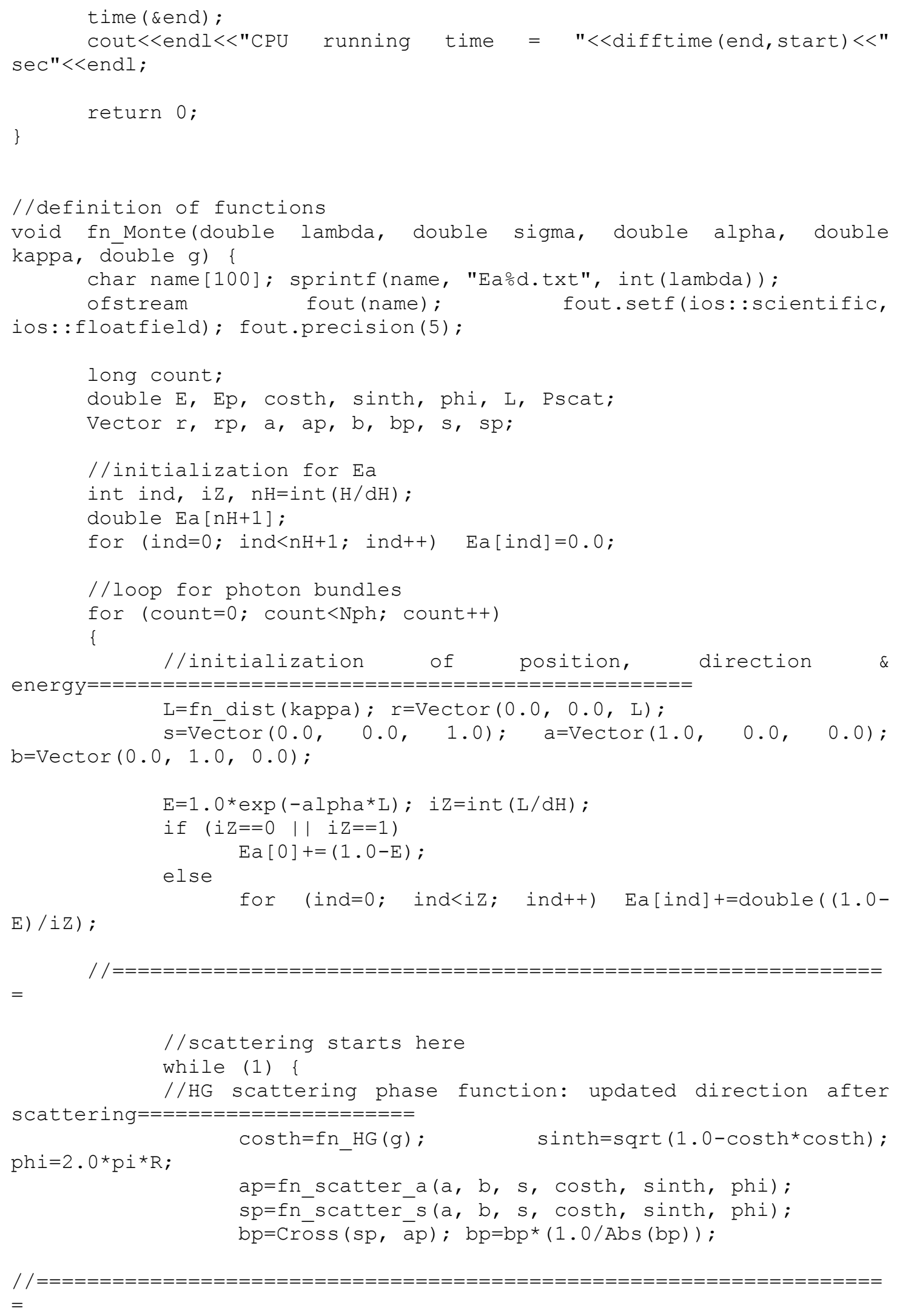




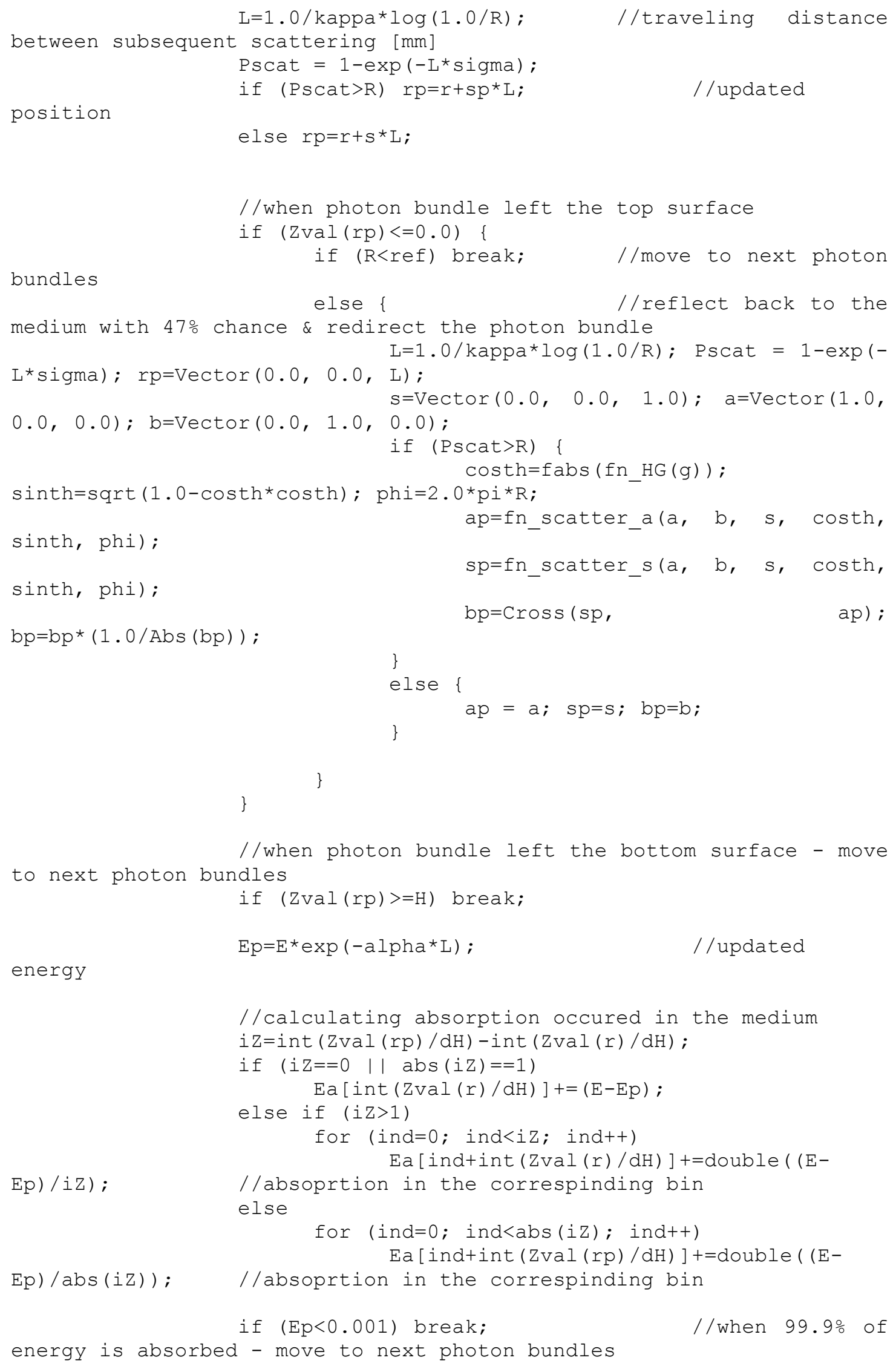




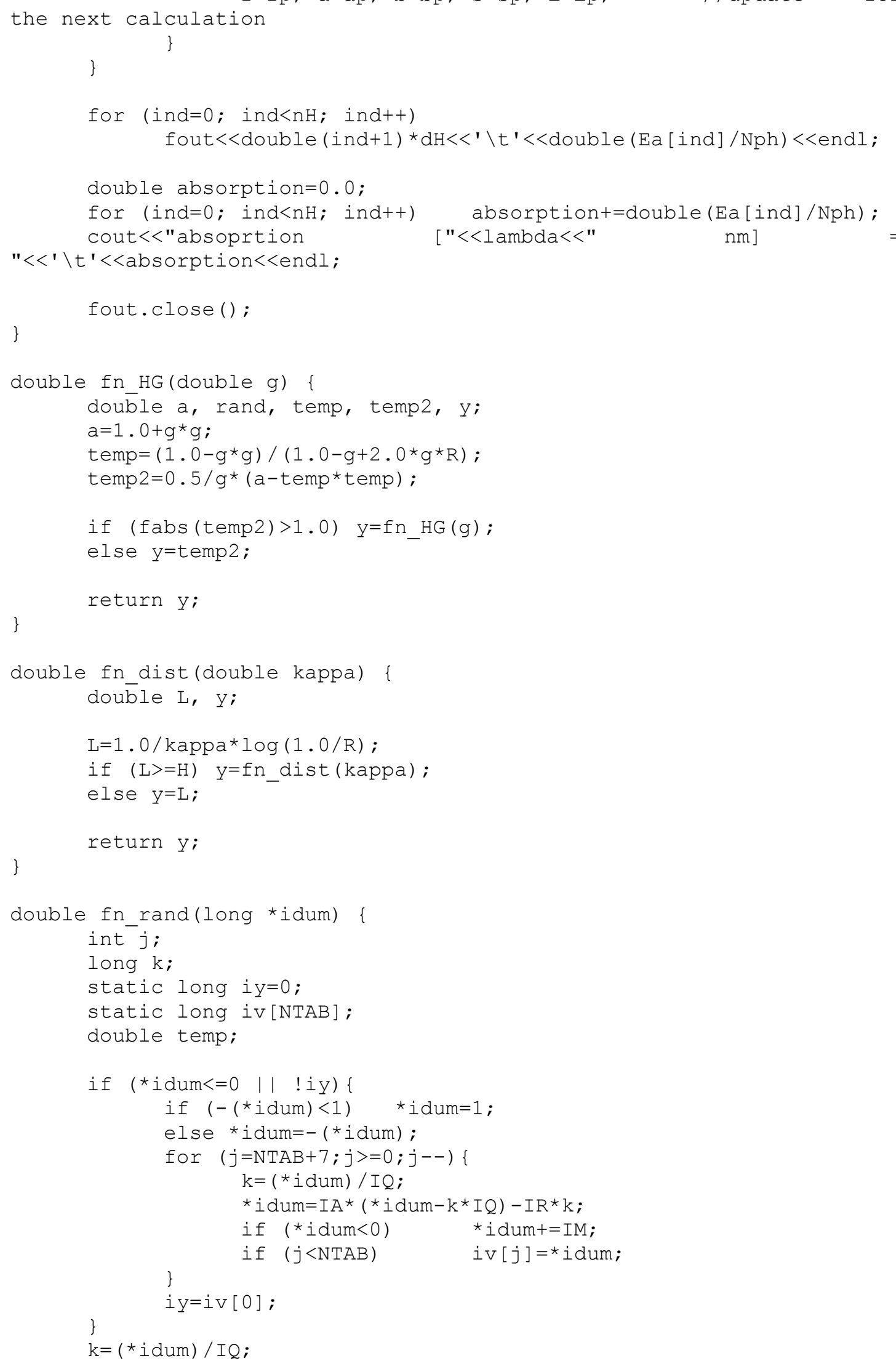




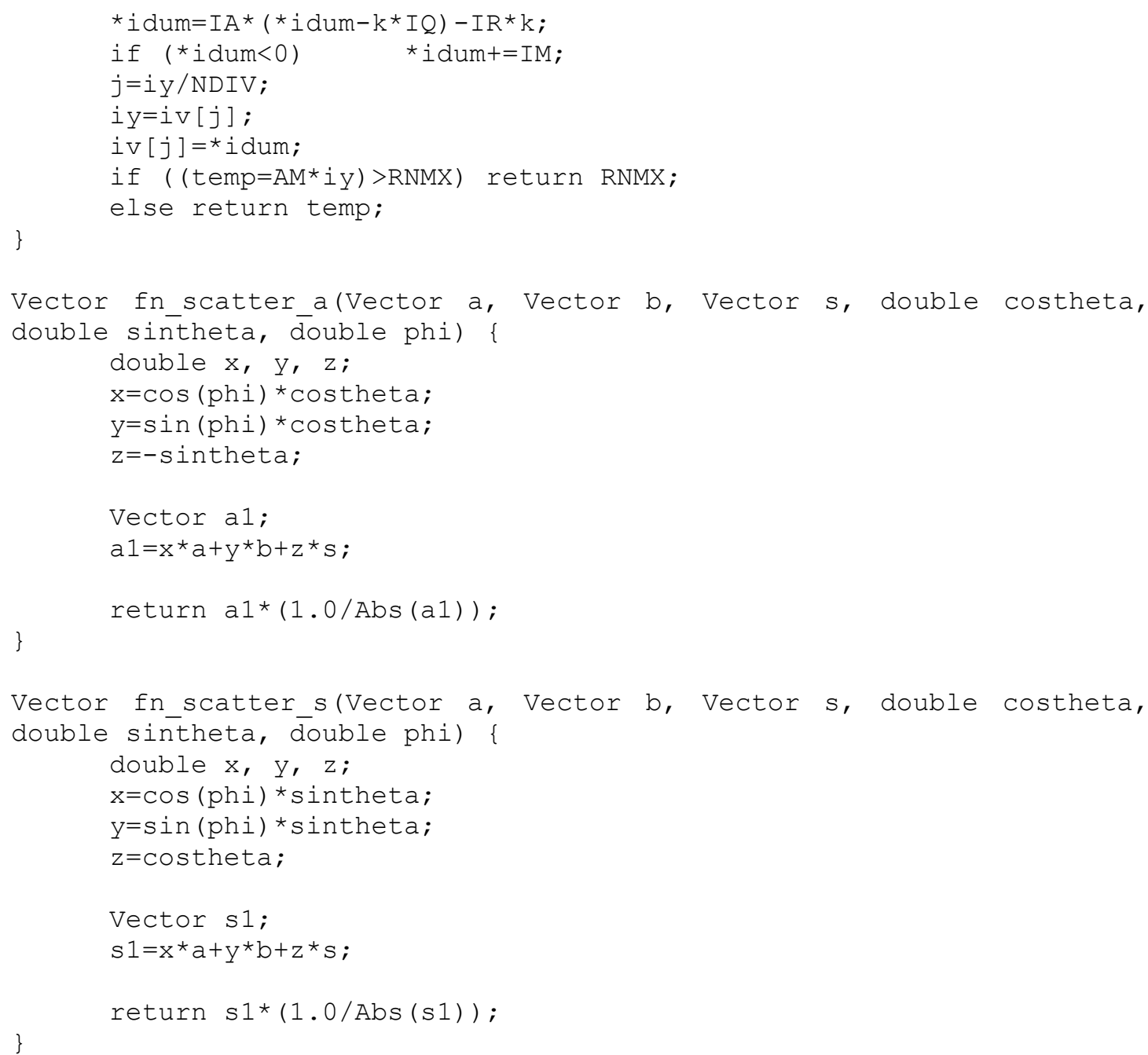

\subsubsection{GNR Absorption Coefficient Conversion \& Penetration Depth Code}

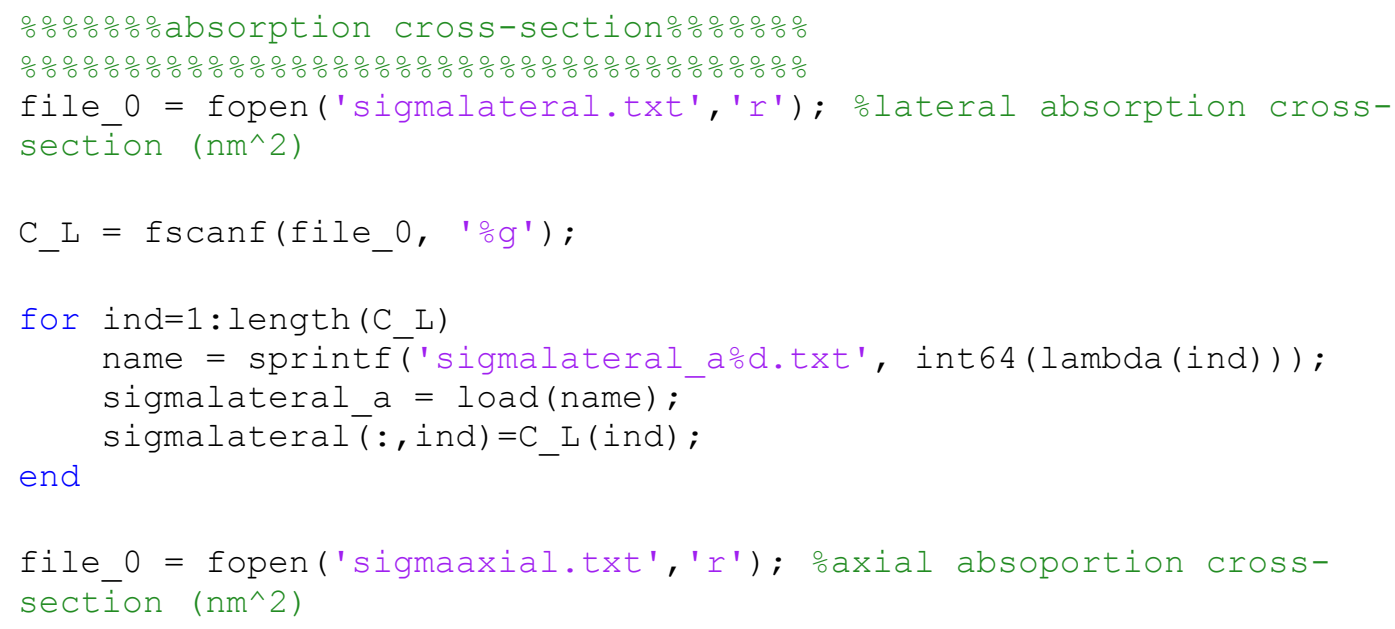




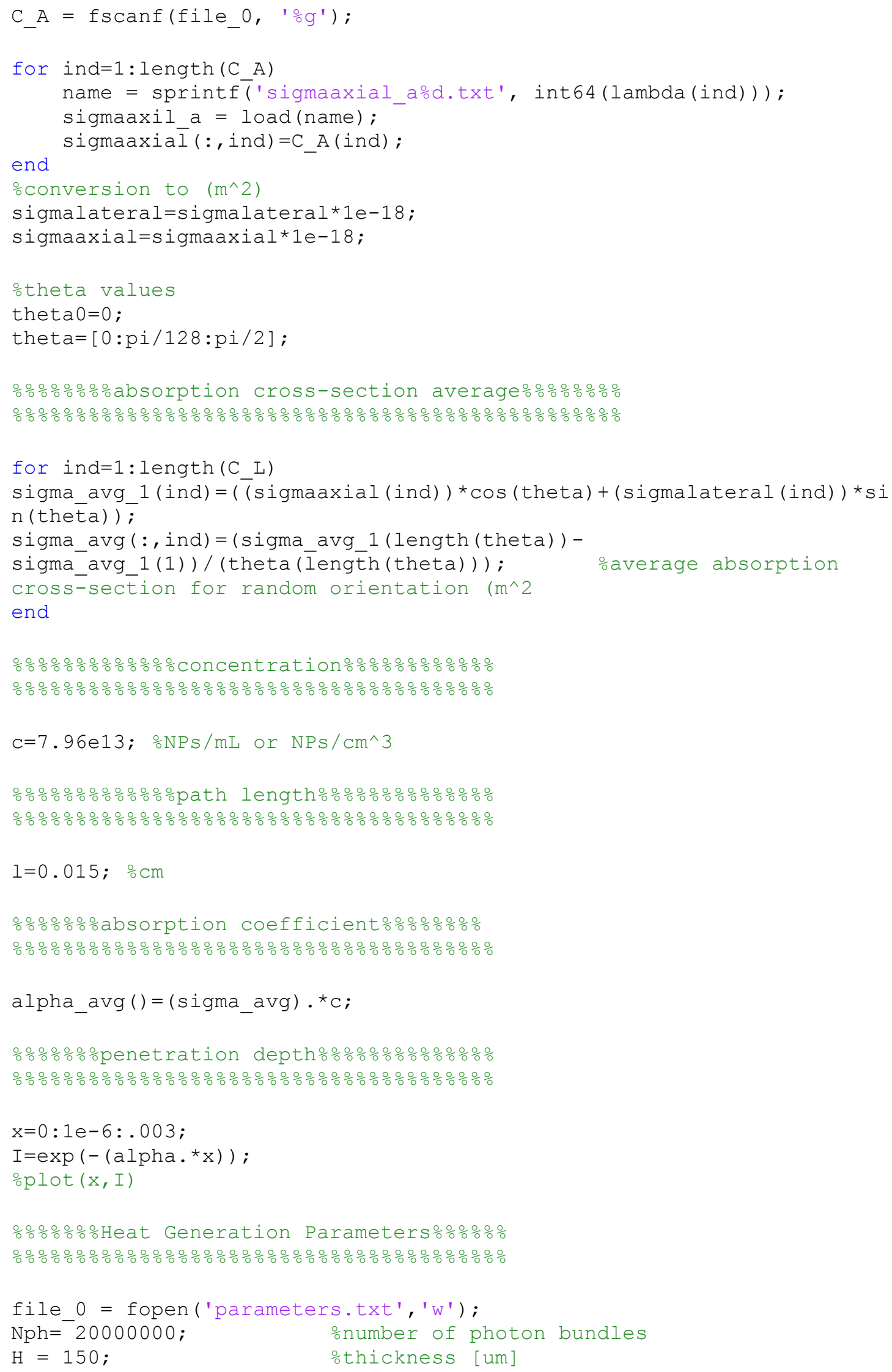




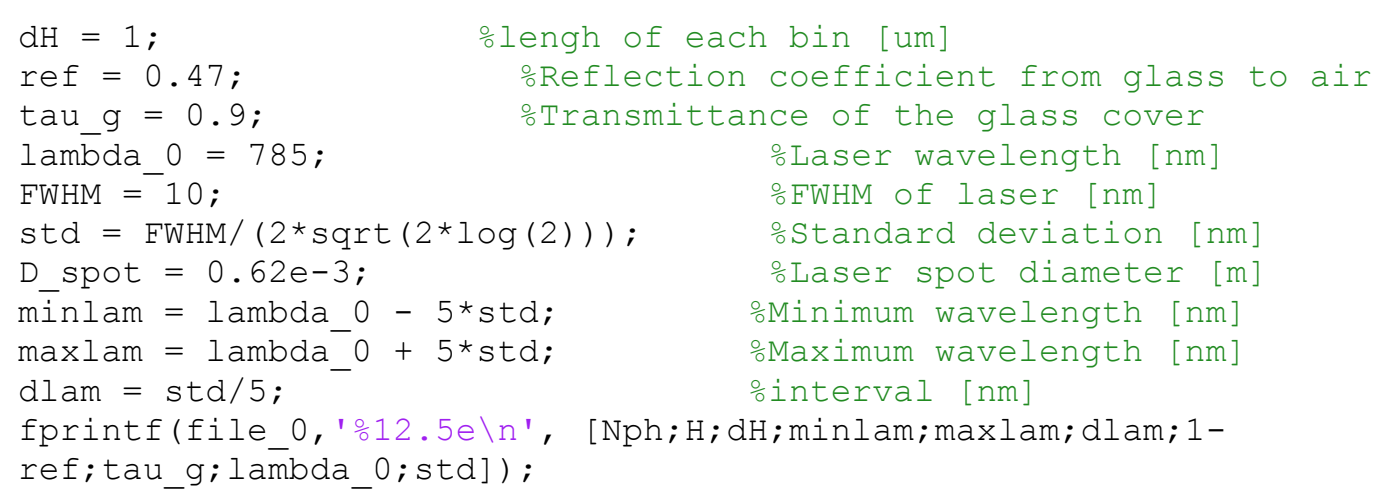

\subsubsection{Nanoparticle Heating Code}

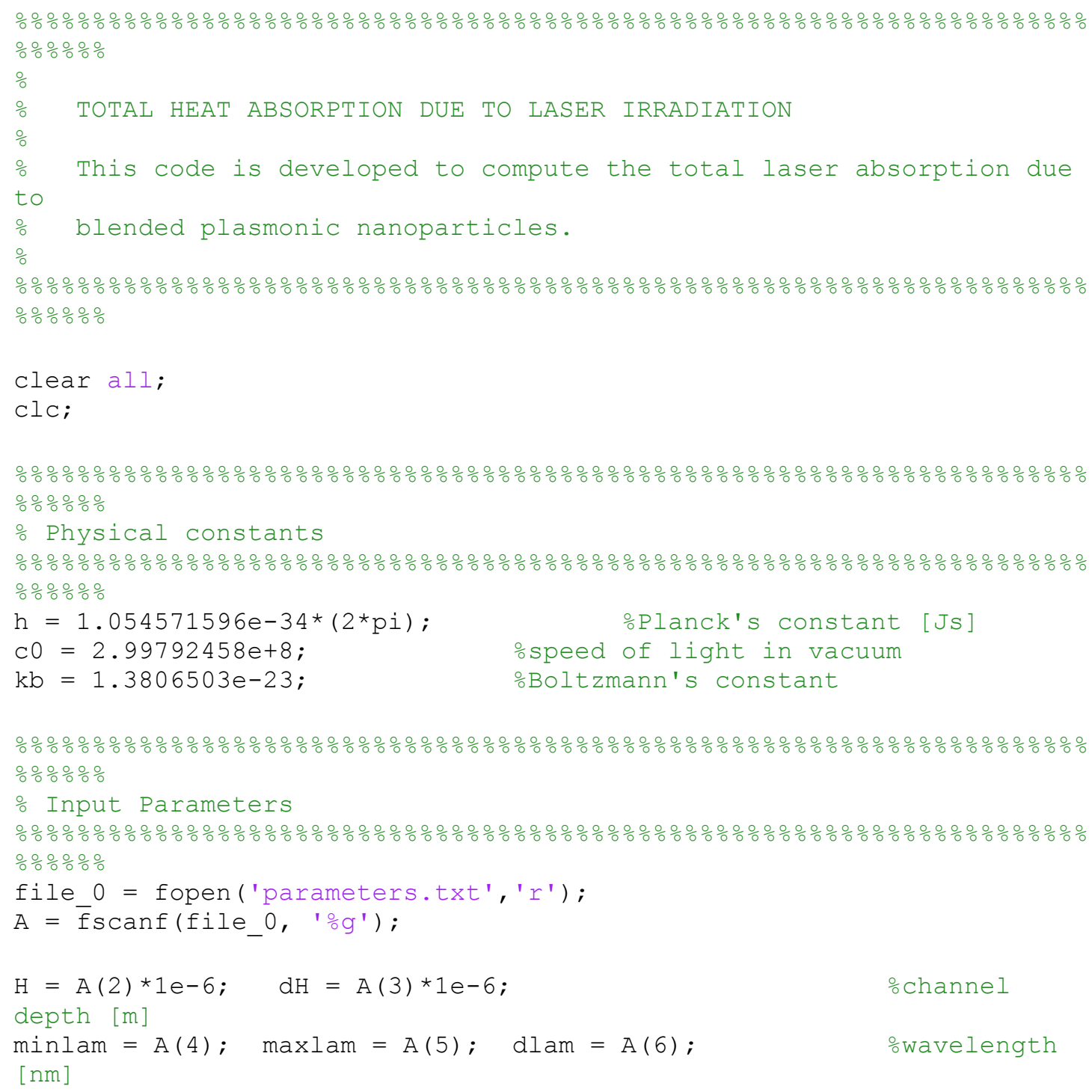




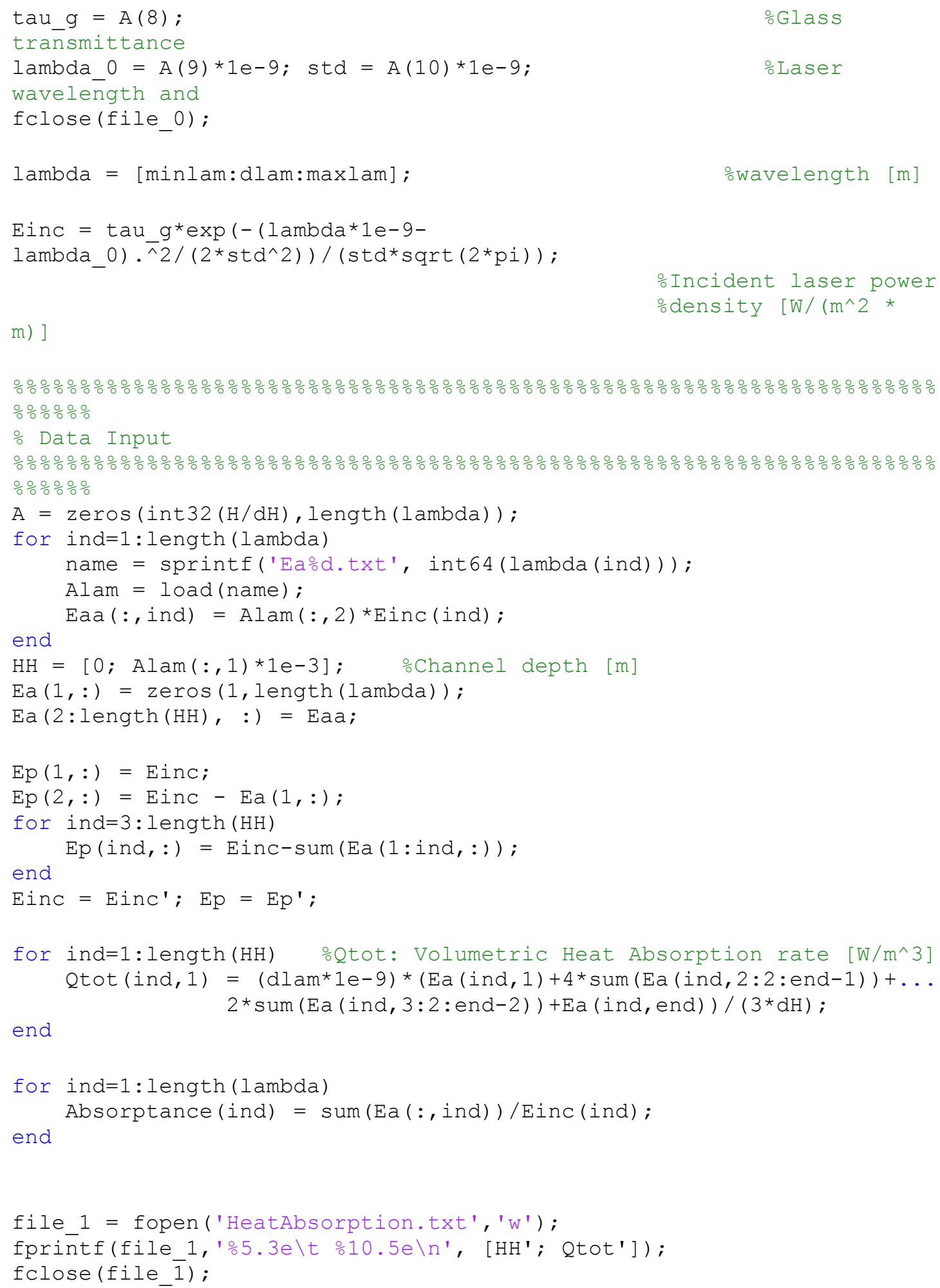




\subsubsection{COMSOL FEA Simulation Code}

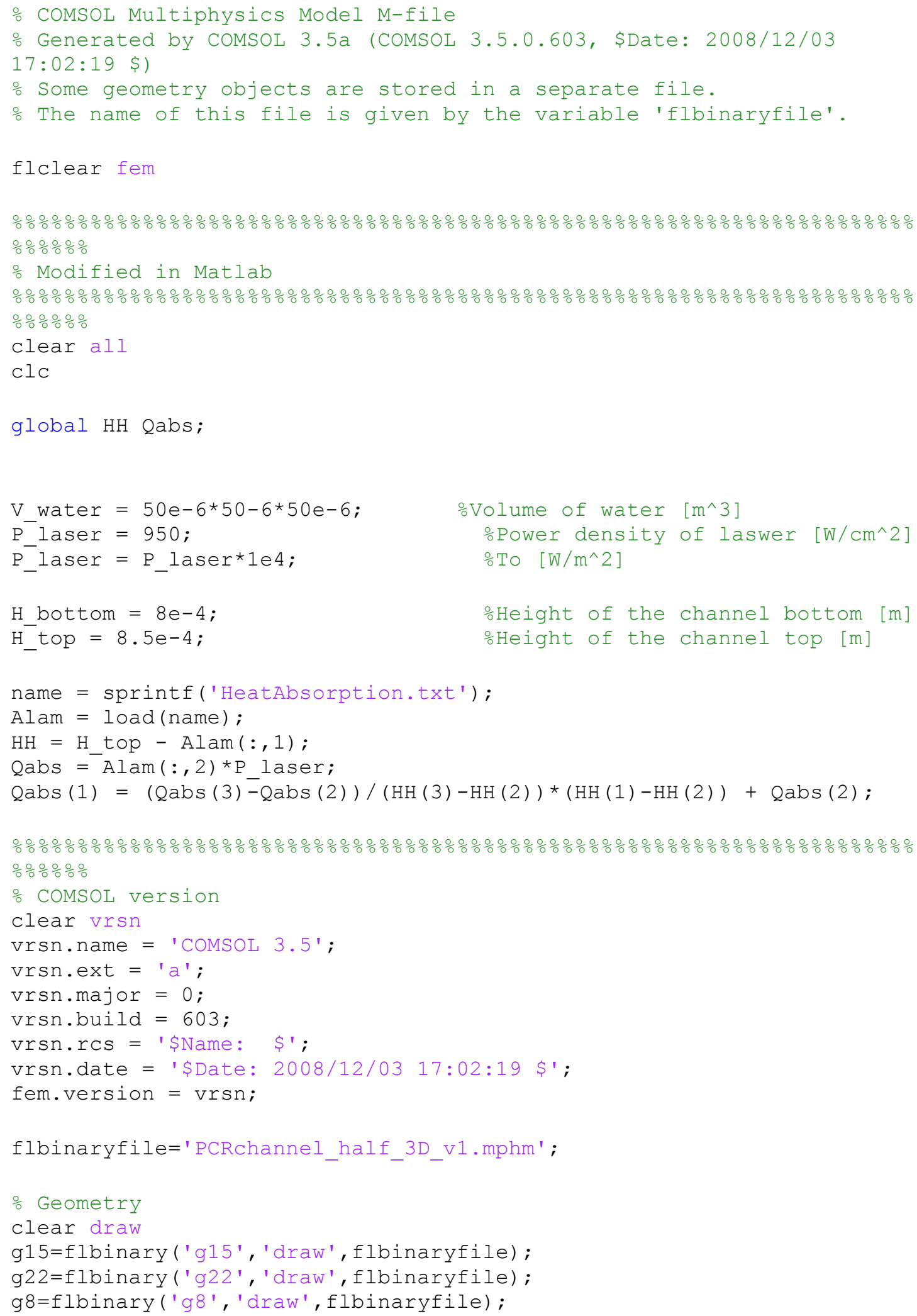




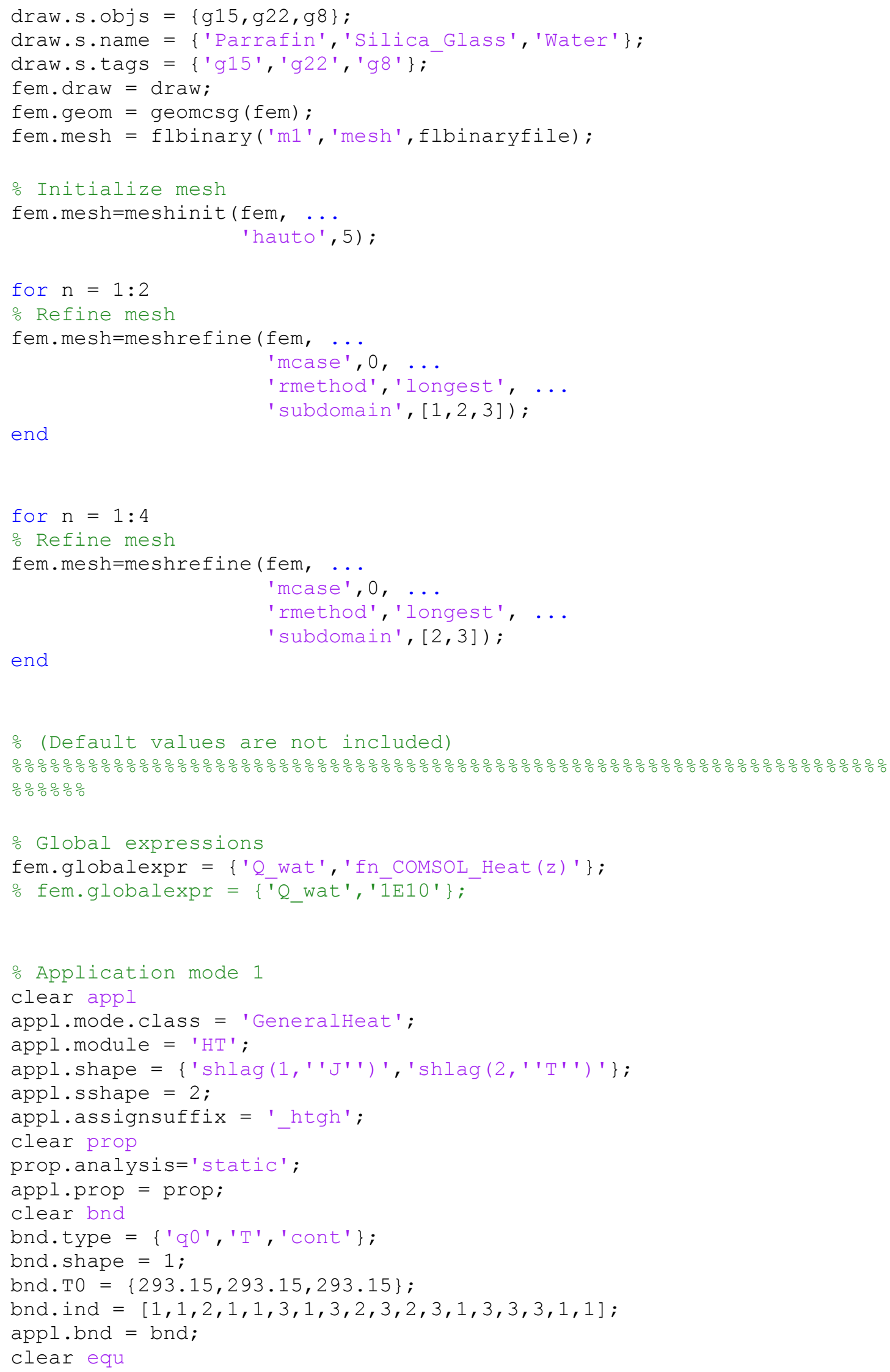




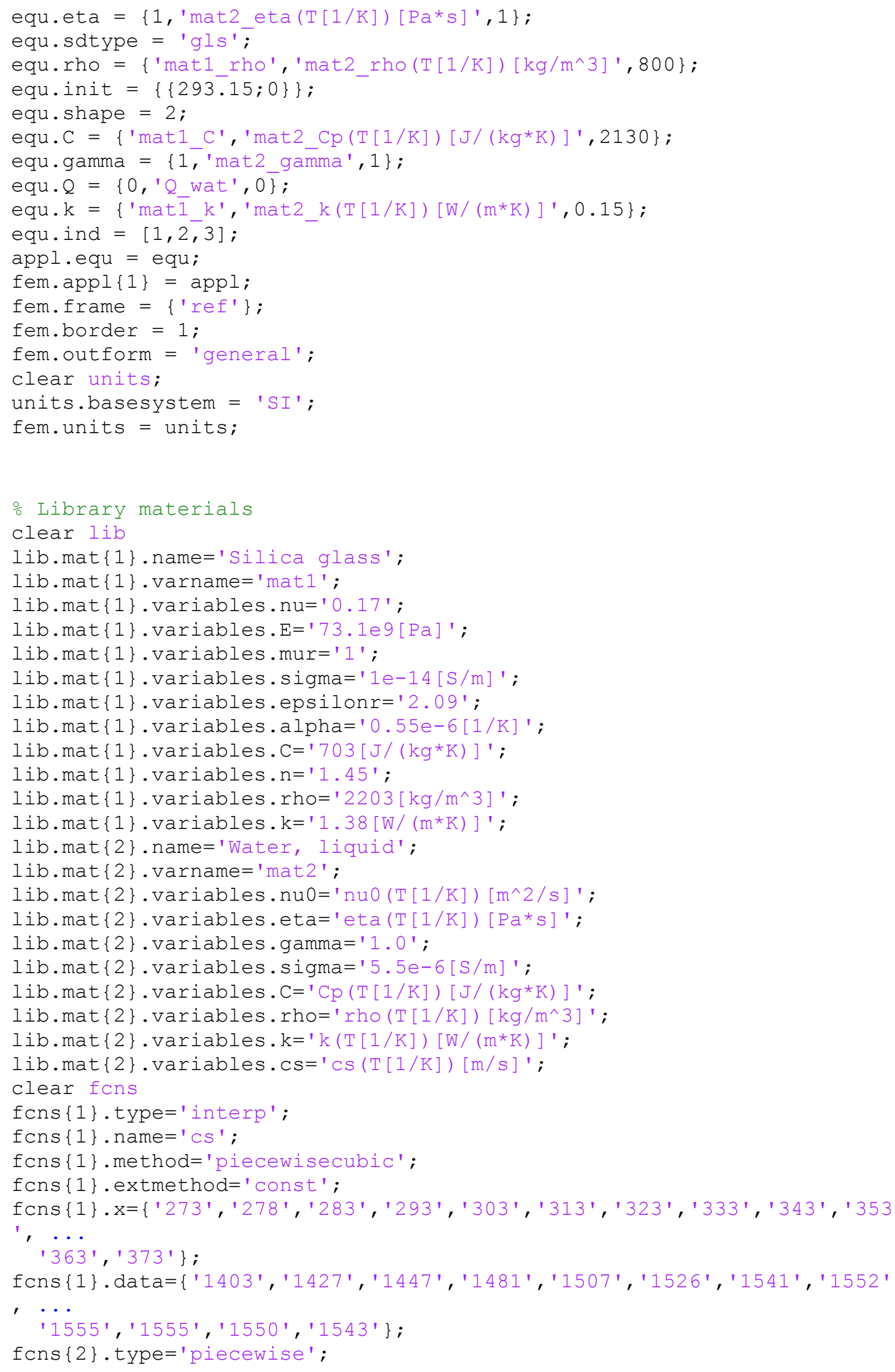




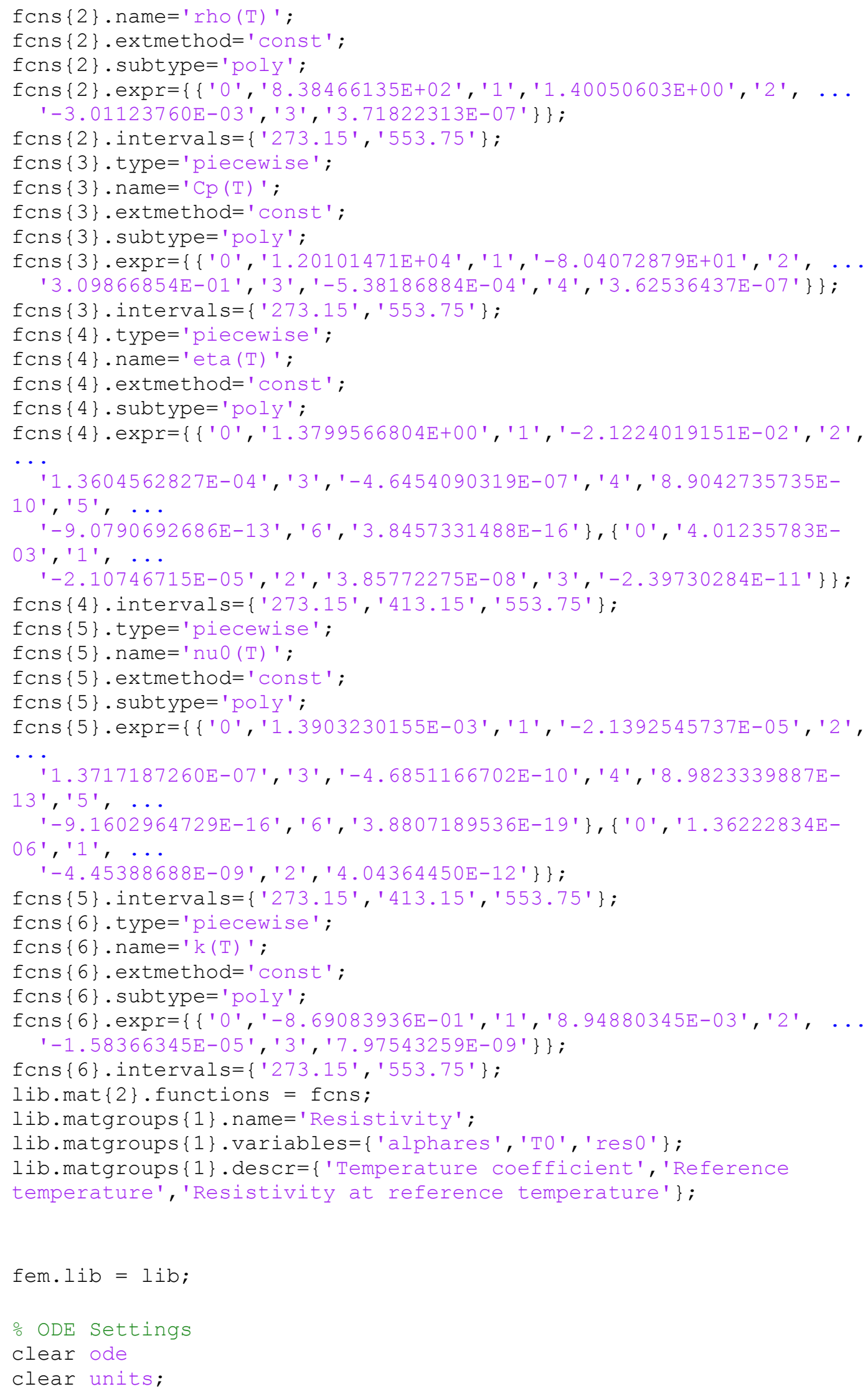




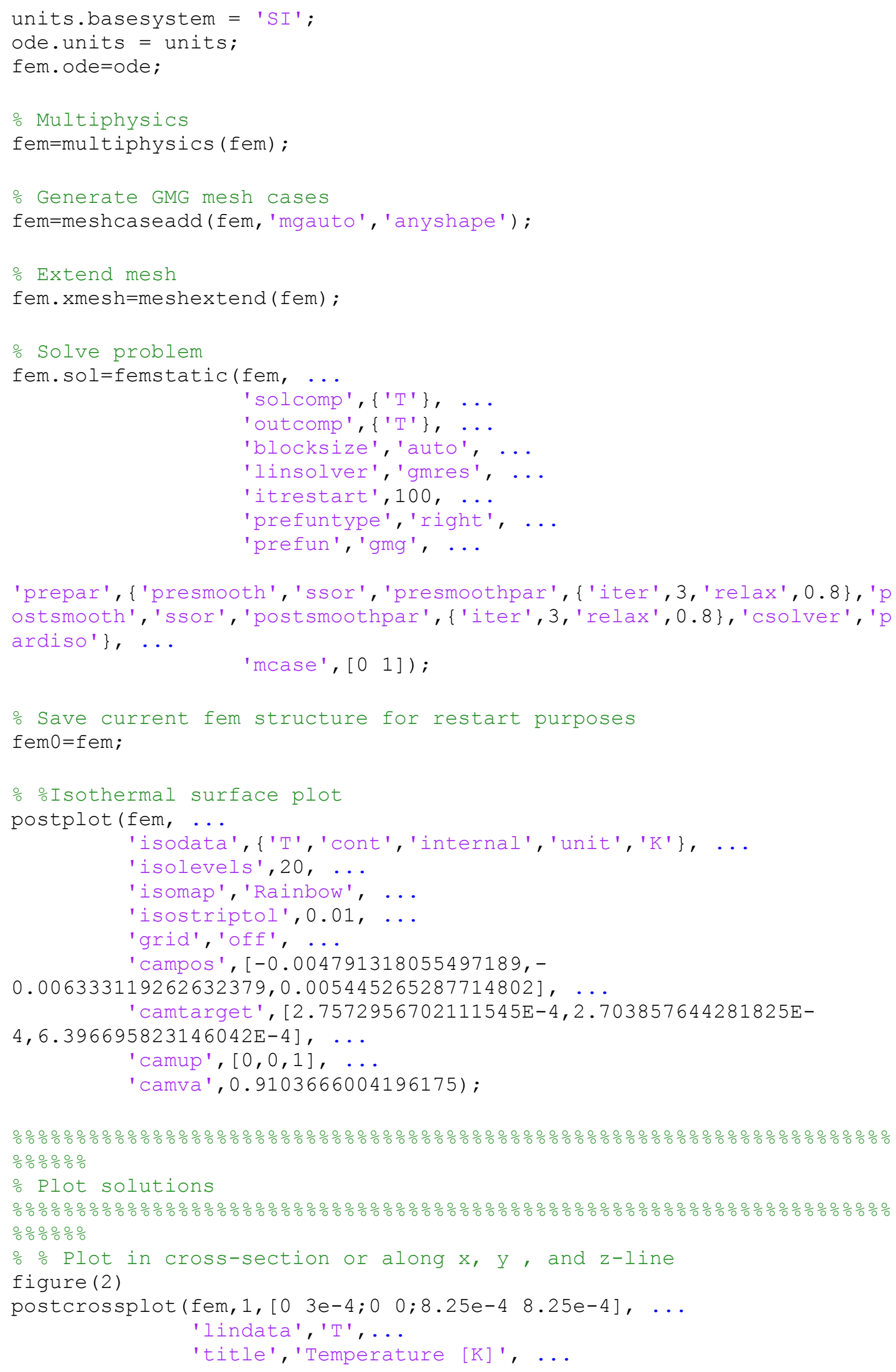




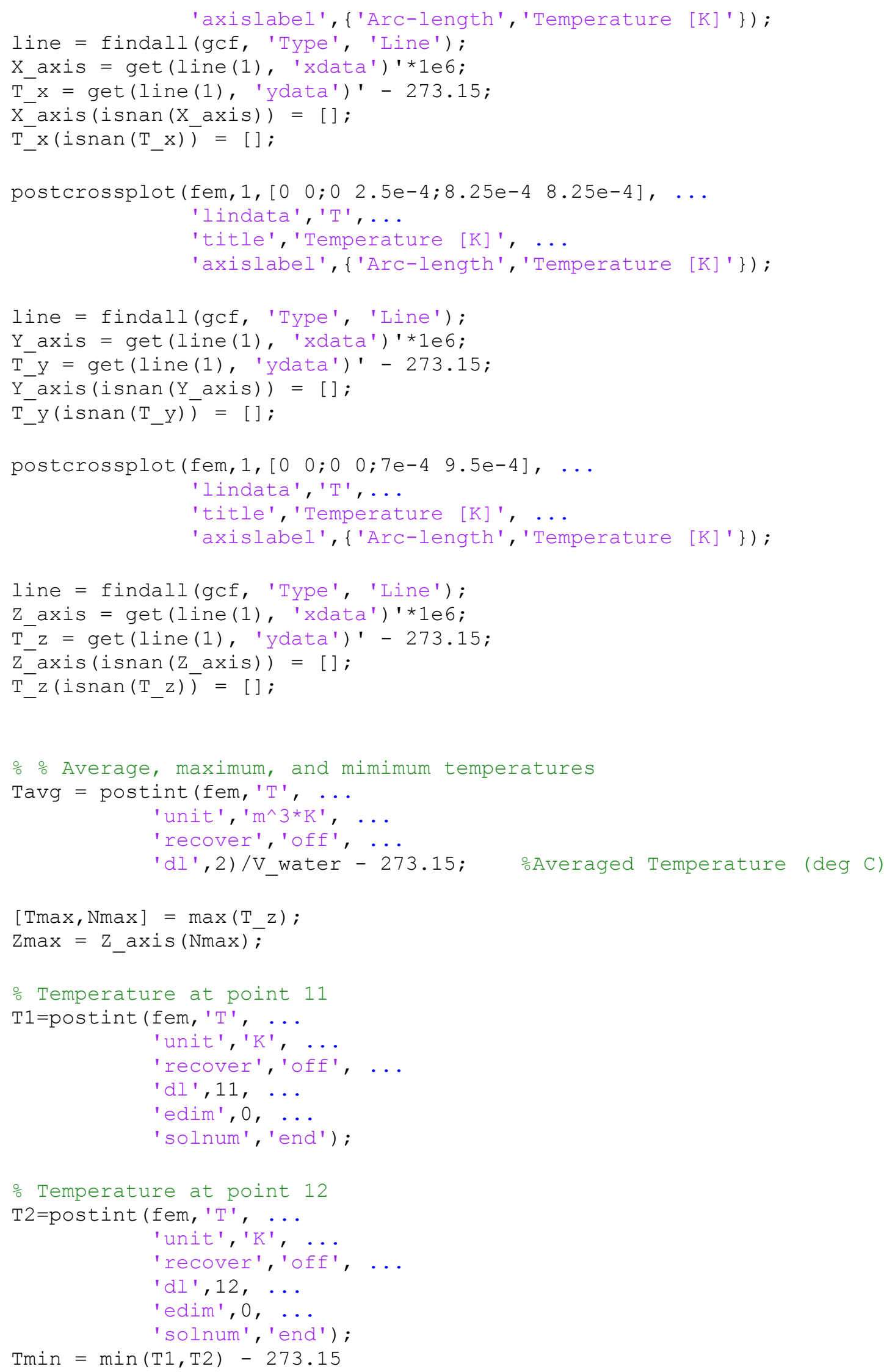




\section{BIBLIOGRAPHY}

[1] Miralles, V., Huerre, A., Malloggi, F., \& Jullien, M.-C. (2013). A Review of Heating and Temperature Control in Microfluidic Systems: Techniques and Applications. Diagnostics (Vol.3,pp.33-67). doi:10.3390/diagnostics3010033

[2] Sharma, V., Park, K., \& Srinivasarao, M. (2009). Colloidal dispersion of gold nanorods: Historical background, optical properties, seed-mediated synthesis, shape separation and self-assembly. Materials Science and Engineering: $R$ : Reports, 65(1-3), 1-38. doi:10.1016/j.mser.2009.02.002

[3] Govorov, A. O., \& Richardson, H. H. (2007). Generating heat with metal nanoparticles We describe recent studies on photothermal effects using colloidal, 2(1), 30-38.

[4] M. Stockman. (2011). Nanoplasmonics: past, present, and glimpse into future. Optics express, 19(20), 22029-106. doi:10.1364/OE.19.022029

[5] Bartlett, J.M.S.; Stirling, D. A short history of the polymerase chain reaction. Meth. Mol. Biol. 2003, 226, 3-6.

[6] Baffou, G., Quidant, R., \& Girard, C. (2009). Heat generation in plasmonic nanostructures: Influence of morphology. Applied Physics Letters, 94(15), 153109. doi:10.1063/1.3116645

[7] Ekici, O., Harrison, R. K., Durr, N. J., Eversole, D. S., Lee, M., \& Ben-Yakar, a. (2008). Thermal Analysis of Gold Nanorods Heated with Femtosecond Laser Pulses. Journal of physics D: Applied physics, 41(18), 185501. doi:10.1088/0022-3727/41/18/185501

[8] Kim, H.; Vishniakou, S.; Faris, G.W. Petri dish PCR: Laser-heated reactions in nanoliter droplet arrays. Lab Chip 2009, 9, 1230-1235.

[9] Oda, R.P.; Strausbauch, M.A.; Huhmer, A.F.; Borson, N.; Jurrens, S.R.; Craighead, J.; Wettstein, P.J.; Eckloff, B.; Kline, B.; Landers, J.P. Infraredmediated thermocycling for ultrafast polymerase chain reaction amplification of DNA. Anal. Chem. 1998, 70, 4361-4368.

[10] Fermer, C.; Nilsson, P.; Larhed, M. Microwave-assisted high-speed PCR. Eur. J. Pharm. Sci. 2003, 18, 129-132.

[11] Issadore, D.; Humphry, K.J.; Brown, K.A.; Sandberg, L.; Weitz, D.A.; Westervelt, R.M. Microwave dielectric heating of drops in microfluidic devices. Lab Chip 2009, 9, 1701-1706. 
[12] Geist, J.J.; Gaitan, M. Microwave power absorption in low-reflectance, complex, lossy transmission lines. J. Res. Natl. Inst. Stand. Technol. 2007, $112,177-189$.

[13] Maltezos, G.; Gomez, A.; Zhong, J.; Gomez, F.A.; Scherer, A. Microfluidic polymerase chain reaction. Appl. Phys. Lett. 2008, 93, 243901:1-243901:3.

[14] Maltezos, G.; Johnston, M.; Taganov, K.; Srichantaratsamee, C.; Gorman, J. Exploring the limits of ultrafast polymerase chain reaction using liquid for thermal heat exchange: A proof of principle. Appl. Phys. Lett. 2010, 97, 264101:1-264101:3.

[15] Chiou, J., Matsudaira, P., Sonin, A., \& Ehrlich, D. (2001). A Closed-Cycle Capillary Polymerase Chain Reaction Machine. Analytical Chemistry, 73(9), 2018-2021. Retrieved from http://dx.doi.org/10.1021/ac001227f

[16] Baffou, G., Quidant, R., \& García de Abajo, F. J. (2010). Nanoscale control of optical heating in complex plasmonic systems. ACS nano, 4(2), 709-16. doi:10.1021/nn901144d

[17] Matsui, T.; Franzke, J.; Manz, A.; Janasek, D. Temperature gradient focusing in a PDMS/glass hybrid microfluidic chip. Electrophoresis 2007, 28, 46064611.

[18] Laval, P.; Lisai, N.; Salmon, J.-B.; Joanicot, M. A microfluidic device based on droplet storage for screening solubility diagrams. Lab Chip 2007, 7, 829-834.

[19] Stroock, A.D.; Dertinger, S.K.W.; Ajdari, A.; Mezic, I.; Stone, H.A.; Whitesides,G.M. Chaotic mixer for microchannels. Science 2002, 295, 647-651.

[20] Adleman, J. R. (2009). Plasmonic Nanoparticles for Optofluidic Applications Thesis by, 2009.

[21] Wu, J.; Cao, W.; Chen, W.; Chang, D.C.; Sheng, P. Polydimethylsiloxane microfluidic chip with integrated microheater and thermal sensor. Biofluidics 2009, 3, 012005:1-012005:7.

[22] Reichert, A., Felbel, J., Kielpinski, M., Urban, M., Steinbrecht, B., \& Henkel, T. (2008). Micro Flow-Through Thermocycler with Simple Meandering Channel with Symmetric Temperature Zones for Disposable PCR-Devices in Microscope Slide Format. Journal of Bionic Engineering, 5(4), 291-298.

[23] Lao, A.I.K.; Lee, T.M.H.; Hsing, I.-M.; Ip, N.Y. Precise temperature control of microfluidic chamber for gas and liquid phase reactions. Sens. Actuator. A 2000, 84, 11-17. 
[24] Dettloff, R., Yang, E., Rulison, A., Chow, A., \& Farinas, J. (2008). Nucleic Acid Amplification of Individual Molecules in a Microfluidic Device. Analytical Chemistry, 80(11), 4208-4213. Retrieved from http://pubs.acs.org/doi/abs/10.1021/ac800339w

[25] Khandurina, J.; McKnight, T.E.; Jacobson, S.C.; Waters, L.C.; Foote, R.S.; Ramsey, J.M. Integrated system for rapid PCR-based DNA analysis in microfluidic devices. Anal. Chem. 2000, 72, 2995-3000.

[26] Taylor, R. a., Phelan, P. E., Otanicar, T., Adrian, R. J., \& Prasher, R. S. (2009). Vapor generation in a nanoparticle liquid suspension using a focused, continuous laser. Applied Physics Letters, 95(16), 161907.

doi:10.1063/1.3250174

[27] Otanicar, T. P., Phelan, P. E., Prasher, R. S., Rosengarten, G., \& Taylor, R. a. (2010). Nanofluid-based direct absorption solar collector. Journal of Renewable and Sustainable Energy, 2(3), 033102. doi:10.1063/1.3429737

[28] Domingues, G., Volz, S., Joulain, K., \& Greffet, J.-J. (2005). Heat Transfer between Two Nanoparticles Through Near Field Interaction. Physical Review Letters, 94(8), 085901. doi:10.1103/PhysRevLett.94.085901

[29] Li, M., Lin, Y.-C., Wu, C.-C., \& Liu, H.-S. (2005). Enhancing the efficiency of a PCR using gold nanoparticles. Nucleic acids research, 33(21), e184. doi:10.1093/nar/gni183

[30] Li, H., Huang, J., Lv, J., An, H., Zhang, X., Zhang, Z., Fan, C., et al. (2005). Nanoparticle PCR: nanogold-assisted PCR with enhanced specificity. Angewandte Chemie (International ed. in English), 44(32), 5100-3. doi:10.1002/anie.200500403

[31] Wang, J., Chen, Y., Chen, X., Hao, J., Yan, M., \& Qiu, M. (2011). Photothermal reshaping of gold nanoparticles in a plasmonic absorber. Optics express, 19(15), 14726-34.http://www.ncbi.nlm.nih.gov/pubmed/21934835

[32] Miao, X., Wilson, B. K., \& Lin, L. Y. (2008). Localized surface plasmon assisted microfluidic mixing. Applied Physics Letters, 92(12), 124108. doi:10.1063/1.2901192

[33] Liu, G. L., Kim, J., Lu, Y., \& Lee, L. P. (2006). Optofluidic control using photothermal nanoparticles. Nature materials, 5(1), 27-32. doi: $10.1038 /$ nmat 1528

[34] Fan, X., \& White, I. M. (2011). Optofluidic Microsystems for Chemical and Biological Analysis. Nature photonics, 5(10), 591-597. doi:10.1038/nphoton.2011.206 
[35] Righini, M., Zelenina, A. S., Girard, C., \& Quidant, R. (2007). Parallel and selective trapping in a patterned plasmonic landscape. Nature Physics, 3(7), 477-480. doi:10.1038/nphys624

[36] Liu, C., \& Li, B. Q. (2011). Computational Multiscattering of Spherical Multilayered Gold Nanoshells. The Journal of Physical Chemistry C, 115(13), 5323-5333. doi:10.1021/jp110252r

[37] Baffou, G., Quidant, R., \& Girard, C. (2009). Heat generation in plasmonic nanostructures: Influence of morphology. Applied Physics Letters, 94(15), 153109. doi:10.1063/1.3116645

[38] Baffou, G., Girard, C., \& Quidant, R. (2010). Mapping Heat Origin in Plasmonic Structures. Physical Review Letters, 104(13), 136805. doi:10.1103/PhysRevLett.104.136805

[40] Baffou, G., Quidant, R., \& Girard, C. (2010). Thermoplasmonics modeling: A Green's function approach. Physical Review B, 82(16), 165424. doi:10.1103/PhysRevB.82.165424

[41] Baffou, G., Quidant, R., \& García de Abajo, F. J. (2010). Nanoscale control of optical heating in complex plasmonic systems. ACS nano, 4(2), 709-16. doi: $10.1021 / \mathrm{nn} 901144 \mathrm{~d}$

[42] Jain, P. K., Lee, K. S., El-Sayed, I. H., \& El-Sayed, M. a. (2006). Calculated absorption and scattering properties of gold nanoparticles of different size, shape, and composition: applications in biological imaging and biomedicine. The journal of physical chemistry. B, 110(14), 7238-48. doi:10.1021/jp057170o

[43] Bohren C F and Huffman D R 1983 Absorption and Scattering of Light by Small Particles. New York:Wiley

[44] Hohenester, U., \& Trügler, A. (2012). MNPBEM - A Matlab toolbox for the simulation of plasmonic nanoparticles. Computer Physics Communications, 183(2), 370-381. doi:10.1016/j.cpc.2011.09.009

[45] García de Abajo, F., \& Howie, A. (2002). Retarded field calculation of electron energy loss in inhomogeneous dielectrics. Physical Review B, 65(11), 115418. doi:10.1103/PhysRevB.65.115418

[46] Lee, B. J., Park, K., Walsh, T., \& Xu, L. (2012). Radiative Heat Transfer Analysis in Plasmonic Nanofluids for Direct Solar Thermal Absorption. Journal of Solar Energy Engineering, 134(2), 021009. doi:10.1115/1.4005756 
[47] Siegel, R., \& Howell, J. R. (2002). Thermal Radiation Heat Transfer. New York: Taylor \& Francis Group.

[48] Han, B., Hanson, W. L., Bensalah, K., Tuncel, A., Stern, J. M., \& Cadeddu, J. a. (2009). Development of quantum dot-mediated fluorescence thermometry $\mathrm{f}$ or thermal therapies. Annals of biomedical engineering, 37(6), 1230-9. doi:10.1007/s10439-009-9681-6

[49] Medintz, I. L., Uyeda, H. T., Goldman, E. R., \& Mattoussi, H. (2005). Quantum dot bioconjugates for imaging, labelling and sensing. Nature materials, 4(6), 435-46. doi:10.1038/nmat1390

[50] Lee, J., \& Kotov, N. A. (2007). Thermometer design at the nanoscale Accurate temperature measurement with high spatial resolution is a, 2(1), 48-51.

[51] Ross, D.; Gaitan, M.; Locascio, L.E. Temperature measurement in microfluidic systems using a temperature-dependent fluorescent dye. Anal. Chem. 2001, 73, 4117-4123.

[52] Garstecki, P., Fuerstman, M. J., Stone, H. a, \& Whitesides, G. M. (2006). Formation of droplets and bubbles in a microfluidic T-junction-scaling and mechanism of break-up. Lab on a chip, 6(3), 437-46. doi:10.1039/b510841a

[53] Pons, T., Medintz, I. L., Sapsford, K. E., Higashiya, S., Grimes, A. F., English, D. S., \& Mattoussi, H. (2007). On the quenching of semiconductor quantum dot photoluminescence by proximal gold nanoparticles. Nano letters, 7(10), 3157-64. doi:10.1021/n1071729+

[54] Dulkeith, E., Morteani, a., Niedereichholz, T., Klar, T., Feldmann, J., Levi, S., van Veggel, F., et al. (2002). Fluorescence Quenching of Dye Molecules near Gold Nanoparticles: Radiative and Nonradiative Effects. Physical Review Letters, 89(20), 203002. doi:10.1103/PhysRevLett.89.203002 Biol. 2003, 226, 3-6.

[55] Hoang, P. H., Park, H., \& Kim, D.-P. (2011). Ultrafast and continuous synthesis of unaccommodating inorganic nanomaterials in droplet- and ionic liquid-assisted microfluidic system. Journal of the American Chemical Society, 133(37), 14765-70. doi:10.1021/ja2054429 